\title{
论文
}

\section{华北克拉通地区有限频体波层析成像 克拉通破坏的空间非均匀性}

\author{
徐小兵 ${ }^{1}$, 赵亮 ${ }^{1,2^{*}}$, 王坤 ${ }^{1}$, 杨建锋 $^{1}$ \\ 1. 中国科学院地质与地球物理研究所岩石圈演化国家重点实验室, 北京 100029 ; \\ 2. 中国科学院青藏高原地球科学卓越创新中心, 北京 100101 \\ * 通讯作者, E-mail: zhaoliang@mail.iggcas.ac.cn
}

收稿日期：2017-05-24; 收修改稿日期：2018-03-13; 接受日期：2018-04-09; 网络版发表日期：2018-07-18 国家重点研发计划项目(编号: 2017YFC0601206)资助

摘要基于国家固定台网389个台站和 10 条流动地震台阵的 832 个宽频地震仪的记录，拾取了纵波在3个频段 $(0.02 \sim 0.1 、 0.1 \sim 0.8$ 和 $0.8 \sim 2.0 \mathrm{~Hz})$ 、来自3 10 个地震事件的 65628 个初至，以及横波在 2 个频段( $0.02 \sim 0.1$ 和 $0.1 \sim 0.8 \mathrm{~Hz}$ )、来自793个地震的 47050 个初至. 在前人和本团队2012年工作的基础上，增加华北克拉通西部数据的 覆盖，考虑地壳校正中入射角的影响，采用有限频多频段联合反演方法，得到华北克拉通上地幔精细的三维纵、 横波速度模型. 新的模型提供了一些新的观测信息和动力学认识: (1) 地幔转换带内的高速异常体表现出非均一 的形态特征，表明俯冲的太平洋板片内部可能发生了尧羽曲变形、断裂，部分物质穿过了 660km间断面; (2) 华北克 拉通东部的上地幔表现出非均匀的小尺度横向速度结构特征，位于南缘和北缘燕山地区的高速异常体可能是残 留的克拉通岩石圈，反映了克拉通破坏的空间非均匀性；(3) 苏鲁造山带下方延伸到 300km的高速异常体构造上 似乎受控于邩庐断裂, 北侧与烟台-青岛-五莲断裂吻合, 可能是北西向俯冲碰撞的扬子克拉通的残留; (4) 华北克 拉通中部的低速异常体存在南北空间差异，北部的低速异常体呈NW-SE走向，向下延伸到地幔转换带，南部的低 速异常体则向下延伸到约200 300km深度; (5) 环绕华北克拉通四周的显生宙造山带、内部古元古代的中央造山 带和郯庐断裂下方是明显的低速异常，低速异常不仅表现出横向上的构造相关性，在60 250km深度内也表现出 垂向上的连贯性. 这个特征暗示岩石圈内固有的构造薄弱带(例如, 碰撞造山缝合带和郯庐断裂)在克拉通破坏中 的重要作用; (6) 本文认为华北克拉通东部破坏表现出的空间非均匀性是太平洋板片俯冲诱导的空间非均匀分布 的湿上升流和克拉通岩石圈内固有的构造薄弱带两个因素共同作用的结果.

关键词上地幔, 地幔转换带, 有限频, 多频段联合反演, 湿上升流, 薄弱带

\section{1 引言}

克拉通的壳幔分异程度较高，岩石圈地幔难熔、
密度较低(吴福元等, 2008), 拥有较厚的岩石圈 (250 300km; Polet和Anderson, 1995; Artemieva和 Mooney, 2001)以及较低的热流(Davies, 2013). 这些特

\footnotetext{
中文引用格式: 徐小兵, 赵亮, 王坤, 杨建锋. 2018. 华北克拉通地区有限频体波层析成像一一克拉通破坏的空间非均匀性. 中国科学: 地球科学, 48: 12231247, doi: 10.1360/N072017-00166

英文引用格式： Xu X B, Zhao L, Wang K, Yang J F. 2018. Indication from finite-frequency tomography beneath the North China Craton: The heterogeneity of craton destruction. Science China Earth Sciences, 61: 1238-1260, https://doi.org/10.1007/s11430-017-9201-y
} 
征使其保持长期的稳定, 然而克拉通属性并非不可改 变, 例如华北克拉通就是经受破坏的典型代表.

华北克拉通位于欧亚板块的东部，是世界上最古 老的克拉通之一，最老的陆壳岩石可追溯到 $3.8 \mathrm{Ga}(\mathrm{Liu}$ 等, 1992). 阴山块体和鄂尔多斯块体在古元古代碰撞, 通过内蒙缝合带拼合成西部陆块(Zhao等, 2003). 稍晚 于此，东、西两个太古宙陆块通过中部造山带碰撞拼 合在一起, $\sim 1.85 \mathrm{Ga}$ 完成克拉通化(Zhao 等, 2001, 2003). 华北克拉通的四周被造山带环绕(图1)：西侧是 早古生代的祁连山造山带(Pan等, 2006; Xiao等, 2009); 北侧是晚二叠-早三叠与西伯利亚克拉通碰撞形成的 中亚造山带(Davis等, 2001; Xiao等, 2003); 南侧和东侧 是在三叠纪与扬子克拉通碰撞形成的秦岭-大别-苏鲁 造山带(Li等, 1993; Zhao等, 2001), 位于华北克拉通东 部边界延伸数千公里的郯庐断裂带的形成、发育与该 中生代碰撞事件有密切关系(Yin和Nie，1996；Zhang， 1997; Zhu等, 2009). 华北克拉通自形成后直到中生代 早期，虽然经历了不同期次的构造事件却依然维持其 稳定状态(赵越等, 2010).

自中生代中期以来华北克拉通发生了构造活化, 东部 200km厚的岩石圈显著减薄到 $80 \mathrm{~km}$, 并发生克 拉通属性的改变, 即克拉通破坏(Griffin等, 1998; Chen 等, 2008; Zheng等, 2008; Zhu和Zheng, 2009; 朱日祥等, 2012). 多学科的证据表明, 西太平洋板片的持续俯冲 是华北克拉通破坏的主导动力因素(朱日祥等，2012). 然而关于克拉通破坏的具体机制尚有争论(吴福元等, 2008，2014)，如岩石圈拆沉作用(邓晋福等，1994; Gao 等, 2004)、热-化学侵蚀作用 $(X u, 2001 ; X u$ 等, 2008)和 橄榄岩-熔体作用(Zhang, 2009)等.

深部的地幔演化过程会引起近地表的地质响应, 例如板片俯冲相关的火山作用(Zhao等，2004; Lei等, 2013)、大陆深俯冲相关的高压-超高压变质岩区 (Guillot等, 2009; Zhao L等, 2017)、板片回撤相关的拉 伸盆地和断裂系的发育(Zhu G等, 2012)等. 为了探究 深部地幔过程的起因和作用，需获取精细的三维地球 内部结构. 层析成像技术是探究地壳和地幔结构的有 效手段之一. 本文的目的正是获取华北克拉通区域上 地幔高分辨率的三维纵、横波速度模型，以期进一步 认识和了解该区域深部地幔的动力学过程.

近年来有许多关于华北克拉通区域的走时成像研 究. 部分模型受分辨率的影响, 局限于大尺度异常的起
因和影响(例如, Lebedev和Nolet, 2003; Huang和Zhao, 2006; Li等，2006，2008; Feng等，2010; Li和van der Hilst, 2010; Obrebski等, 2012; Zhao等, 2012, Chen等, 2015): 受限于面波成像的约束和低频的数据成份, Lebedev和Nolet(2003) 结果的横向分辨率 $\geq 500 \mathrm{~km}$; Obrebski等(2012)的横向分辨率为 $3^{\circ} \sim 4.5^{\circ}$; $\mathrm{Li}$ 等 $(2006$, 2008)、Li和van der Hilst(2010)的最高分辨率为 $\sim 300 \mathrm{~km}$; Feng等(2010)横向分辨率为浅部的 2 到深部 的 $4^{\circ}$; Zhao等 $(2012)$ 则为 $\geq 200 \mathrm{~km}$. 部分模型则关注于 特定的研究区域, 如苏鲁造山带的研究 $(X u$ 等, 2002), 北京周边区域地壳的研究(Huang和Zhao, 2004; Lei等, 2008); Sun等(2006, 2008a, 2008b)得到地壳和地幔最顶 部的速度结构, 显示出和地表构造很好的一致性, 却未 探讨浅部结构与深部过程的联系.

与利用Zhao等(1994)的射线层析成像方法在华北 克拉通区域的研究不同(例如, Huang和Zhao, 2006; Tian等，2009; Tian和Zhao，2011; Xu和Zhao，2009; Lei，2012)，本文采用有限频灵敏度核函数方法(Dahlen等，2000; Hung等，2000)对整个华北克拉通区域 $\left(100^{\circ} \sim 124^{\circ} \mathrm{E} 、 26^{\circ} \sim 46^{\circ} \mathrm{N}\right)$ 进行成像研究. 对比本团队 2012年的工作(Zhao等, 2012), 本文增加了华北克拉通 西部数据的覆盖; 对地震数据分频段滤波拾取初至后, 采用了有限频多频段联合反演方法进行成像; 并在地 壳校正中考虑了入射角的影响. 分辨率测试表明，新 的速度模型具有更高的空间分辨率，不仅在整体特征 上和前人的工作一致，也揭示了华北克拉通破坏的空 间非均匀性以及克拉通岩石圈内部构造在克拉通破坏 过程中的重要作用.

\section{2 数据与方法}

\section{1 研究区域与观测系统}

本文基于 $100^{\circ} \sim 124^{\circ} \mathrm{E} 、 26^{\circ} \sim 46^{\circ} \mathrm{N}$ 区域范围内多个 台阵观测的地震数据. 其中包括本团队2012年层析成 像工作所采用的地震观测数据: (1) 华北深部结构探 测计划(NCISP)1 8期, 共681个台, 累计观测周期为 2000 2011年(图1绿色三角形); (2) 中国国家地震台网 (Zheng等, 2010), 共389个台, 观测周期为2007 2012年 (图1粉色圆圈). 此外, 本文还增加了北京大学布设的 流动台阵(共100台，观测周期为2005 2010年，图1红 色三角形)以及中国地震局地球物理研究所布设的流 


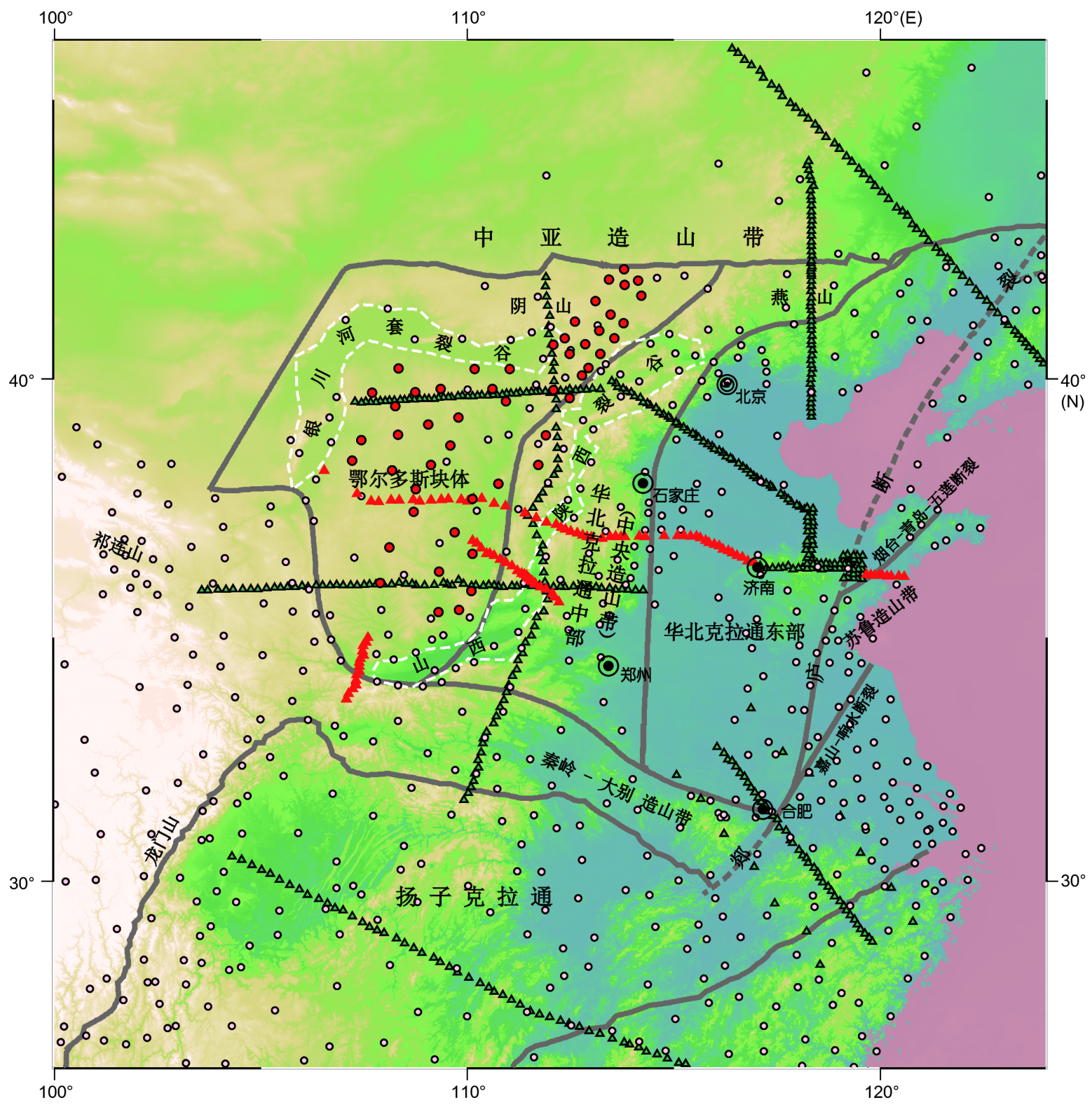

图 1 华北克拉通区域构造及地震台站位置分布图

灰色实线表示主要构造单元的界限; 灰色的虚线表示郯庐断裂; 白色的虚线表示新生代裂谷系; 粉色的圆圈是中国国家台网中心布设的固定 台站; 绿色的三角是中国科学院地质与地球物理研究所布设的流动地震台阵NCISP(1 8期); 红色的三角是北京大学布设的流动地震台阵; 红 色的圆圈是中国地震局地球物理研究所布设的流动地震台阵

动台阵(共51台, 观测周期为2010 2011, 图1红色圆圈). 相对而言，华北克拉通西部地区以及华北盆地地区的 射线覆盖变得更加密集.

\section{2 数据的拾取与分析}

对于远震体波，选择震中距在 $25^{\circ} \sim 98^{\circ}$, 初至到时 拾取数目 $>10$ 的地震事件. 为保证地震-台站对的空间
均匀性, 去除部分震源位置相对重复的地震事件. 纵 波数据体最终选用 310 个地震事件(网络版附图 $1 \mathrm{a}$, http://earthcn.scichina.com), 横波数据体最终选用793 个地震事件(网络版附图1b). 地震波的散射和波前愈 合现象会导致初至到时存在着频率相关性(网络版附 图2a; Hung等, 2004; Zhao等, 2016). 为利用这一特性, 对纵波和横波数据体分别选用不同频段的带通滤波器 
滤波、拾取初至(网络版附图2b; 纵波选用0.02 0.1、 $0.1 \sim 0.8$ 和 $0.8 \sim 2.0 \mathrm{~Hz}$ 频段; 横波选用 $0.02 \sim 0.1$ 和 $0.1 \sim 0.8 \mathrm{~Hz}$ 频段). 基于IASP91模型假设(Kennett和Engdahl, 1991), 采用多道互相关方法(Van Decar和Crosson, 1990)计算相对走时残差. 为保证反演数据的可靠 性，通过纵、横波的相对走时残差比值去除完全偏离 参考斜率的走时奇异数据(网络版附图 $3 a$ ). 最终共拾 取 65628 个纵波初至到时，均方根误差 $0.004 \mathrm{~s}$ 和 47050 个横波初至到时, 均方根误差 $0.012 \mathrm{~s}$ (表 1 ). 通过与其他 自动拾取走时的方法对比(例如, Chevrot等, 2014), 本 文所用的方法由于加入了交互确认，可以有效地减少 周期跳跃(cycle skip)的情况，因此具有更小的拾取误 差. 台站在不同后方位角间距内的平均走时残差值直 接显示出纵、横波波速横向上的变化(网络版附图 3b d), 也反映出最终成像速度模型的一阶近似特征: 鄂尔多斯块体呈现负的走时残差(实际到时较理论到 时早), 中央造山带的北缘(大同火山区域)则呈现正的 走时残差. 这两个区域的走时残差值并不表现出后方 位角的相关性.

\section{3 方法}

本文采用有限频体波走时层析成像方法(Dahlen 等, 2000; Hung等, 2000, 2004; Ren和Shen, 2008; Liang 等，2011)反演华北克拉通下方纵、横波速度结构，且 基于IASP91一维层状模型计算三维灵敏度核函数. 相 比于传统的射线理论，有限频灵敏度核函数方法考虑 了波前愈合、散射以及其他衍射的影响，地震波的走 时与射线路径周围的三维速度结构有关(Dahlen等, 2000), 可表示为

$$
\delta t=\iiint_{\oplus} K(\mathbf{x}) \delta c(\mathbf{x}) / c(\mathbf{x}) \mathrm{d}^{3} \mathbf{x},
$$

式中, $K$ 是走时残差 $\delta$ 对应的三维灵敏度核函数, $\delta c(\mathbf{x}) /$ $c(\mathbf{x})$ 是需要成像的速度扰动解. 旁轴近似下, 考虑第一 菲涅尔带内速度结构的影响，灵敏度核函数可以表述 为(Dahlen等, 2000):

$$
K=\frac{1}{2 \pi c}\left(\frac{R}{c_{\mathrm{r}} R^{\prime} R^{\prime \prime}}\right) \frac{\int_{0}^{\infty} \omega^{3}\left|\mathrm{~s}_{\mathrm{syn}}(\omega)\right|^{2} \sin (\omega \Delta T) \mathrm{d} \omega}{\int_{0}^{\infty} \omega^{2}\left|\mathrm{~s}_{\mathrm{syn}}(\omega)\right|^{2} \mathrm{~d} \omega},
$$

式中, $\Delta T$ 是“源点-散射点-接收点”散射路径与“源点-接 收点”直达路径的走时差; $R 、 R^{\prime}$ 和 $R^{\prime \prime}$ 分别是直达路
径、前向的源-散射点路径和后向的接收点-散射点路 径上的射线几何传播因子; $\left|\mathrm{s}_{\mathrm{syn}}\right|^{2}$ 是与拾取走时残差过 程中滤波频段相关的理论地震记录的能谱. 如 2.2 节所 述, 对于相同的震相初至, 走时残差的拾取与滤波频段 相关(网络版附图 2a), 这是由于不同频率的灵敏度核 函数 $K$ 对射线路径周围速度结构的空间采样范围的差 异造成的. 因此，对于每个源-台对在不同频段内拾取 走时残差将会提供更多的空间采样信息.

研究区域被划分为 $64 \times 64 \times 64$ 个三维网格，中心位 于 $\left(112^{\circ} \mathrm{E}, 36^{\circ} \mathrm{N}\right)$, 经度向步长 $42 \mathrm{~km}$ ，纬度向步长 $\sim 35 \mathrm{~km}$, 深度向步长 $23 \mathrm{~km}$. 模型参数化后, 走时方程 可以写成离散形式:

$$
\mathbf{d}_{i}=\mathbf{G}_{i j} \mathbf{m}_{j},
$$

式中, $\mathbf{d}_{i}$ 是第 $i$ 个观测走时; $\mathbf{G}_{i j}$ 是第 $i$ 个观测走时的三维 灵敏度核函数在网格节点 $j$ 处一定空间范围的体积分; $\mathbf{m}_{j}$ 是第 $j$ 个节点处的模型参数. 反演问题的阻尼最小二 乘解(Paige和Saunders, 1982; Nolet, 1985)为

$$
\widehat{\mathbf{m}}=\left(\mathbf{G}^{\mathrm{T}} \mathbf{G}+\theta^{2} \mathbf{I}\right)^{-1} \mathbf{G}^{\mathrm{T}} \mathbf{d},
$$

式中, $\mathbf{I}$ 是单位矩阵; $\theta$ 是兼顾考虑走时数据的方差降和 模型范数选取的最优反演阻尼因子(图2). 为了抑制射 线空间采样非均匀性在反演中引入的噪音扰动, 除了 简单的范数阻尼, 对矩阵 $\mathbf{G}$ 施加卷积平滑因子(Meyerholtz等, 1989; Hung等, 2004). 方程(4)可改写为

$$
\widehat{\mathbf{m}}=\mathbf{W}\left(\mathbf{W}^{\mathrm{T}} \mathbf{G}^{\mathrm{T}} \mathbf{G} \mathbf{W}+\theta^{2} \mathbf{I}\right)^{-1} \mathbf{W}^{\mathrm{T}} \mathbf{G}^{\mathrm{T}} \mathbf{d},
$$

式中, $\mathbf{W}$ 是卷积平滑因子, 本文纵、横波选取的经、纬 向和深度向的互相关长度均为 2 倍网格距. 本文讨论的 纵波速度模型选用的阻尼因子为 40.0 , 相应的走时数 据方差降为 $73 \%$ (图2蓝色圆圈所示); 横波速度模型选 用的阻尼因子为 50.0 , 相应的走时数据方差降为 $71 \%$ (图2红色三角所示).

\section{4 地壳非均匀结构校正}

受地壳中低速沉积盖层的影响, 远震体波近垂直 入射到地表接收点, 射线在地壳内交叉很少, 对地壳 的约束不足, 导致在地壳深度范围内分辨率较低. 因 此在反演过程中, 地壳结构的非均匀性引起的走时差 通常被反演算法归结到地幔波速结构中，这种现象称 为地壳非均匀性结构对成像结果的“拖尾效应”. 这种 

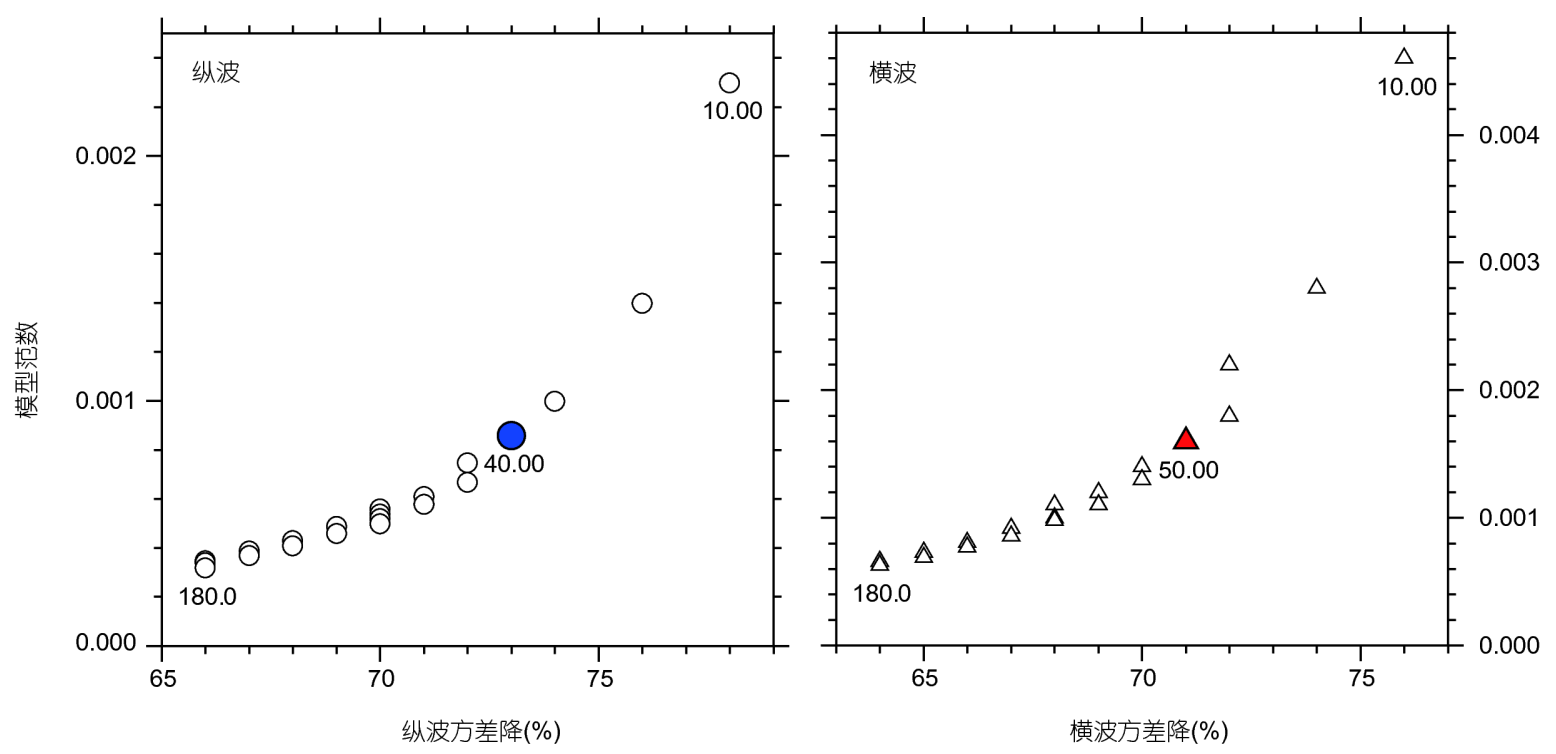

图 2 纵波和横波的模型范数-方差降图(折衷曲线)

圆形和三角形分别代表纵波和横波的折衰曲线. 蓝色圆形和红色三角形分别代表图 4 和 5 的模型, 相应的纵波方差降为 $73 \%$, 横波方差降为 $71 \%$
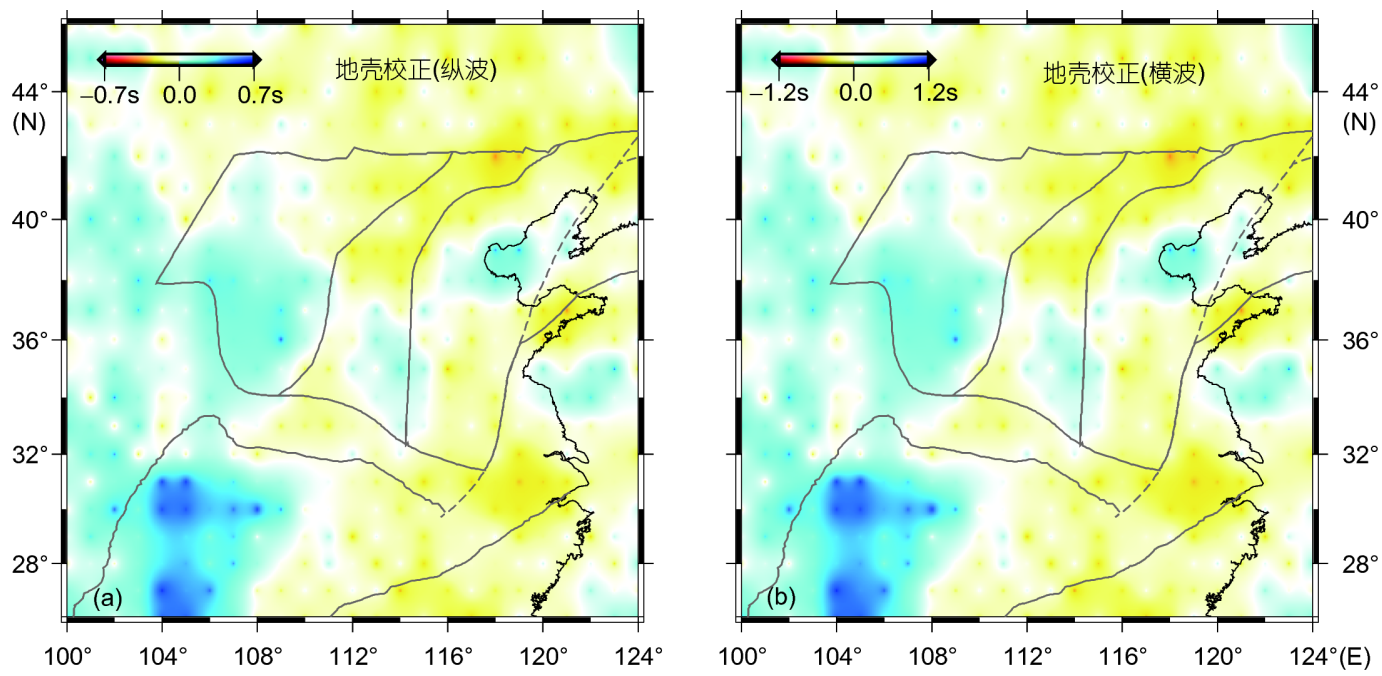

图 3 纵波和横波的地壳校正量

校正模型采用Sun等(2008a)的区域地壳横波速度模型, 纵波速度模型通过平均泊松比 1.765 获得. 纵、横波的地壳校正深度均为 $37.6 \mathrm{~km}$

现象在台站稀疏的区域显得相对明显．对于地壳波速 结构复杂的地区，消除地壳非均匀性结构的影响，能 够显著提高地幔速度结构成像结果的可靠性(Ekström 和Dziewonski, 1998; Allen等, 2002; Li等, 2006; Koulakov等, 2009). 对于华北, 需要减少该区域复杂的地壳 非均匀性结构对最终的速度模型的影响: (1) 从观测的 走时残差中扣除由区域地壳速度模型(Sun等，2008a)
计算的地壳走时异常(图3). Sun等(2008a)的地壳速度 模型厚度从 $30.76 \mathrm{~km}$ 变化到 $61.84 \mathrm{~km}$, 由于缺少上地幔 顶部的速度模型, 本文选择的地壳校正深度为 $30.76 \mathrm{~km}$. 同时，在反演中去除顶部 $30.76 \mathrm{~km}$ 内的灵敏 度核函数的计算. 在本团队2012年工作基础上，根据 IASP91模型计算理论地震射线的入射角, 基于Sun等 (2008a)的地壳速度模型从地表向下直至校正深度逐 
表 1 相对走时残差数据信息 ${ }^{\mathrm{a})}$

\begin{tabular}{cccccc}
\hline 震相 & 走时数据体参数 & $0.02 \sim 0.1 \mathrm{~Hz}$ 频段 & $0.1 \sim 0.8 \mathrm{~Hz}$ 频段 & $0.8 \sim 2.0 \mathrm{~Hz}$ 频段 & 合计 \\
\hline 纵波 & 走时数目 & 19681 & 28755 & 17192 & 65628 \\
$\begin{array}{c}\text { (互相关系数 }>0.85) \\
\text { 310个地震事件 }\end{array}$ & 互相关系数 & 0.97 & 0.93 & 0.92 & 0.94 \\
\hline 枃方根误差 $(\mathrm{s})$ & 0.0062 & 0.00036 & 13175 & 0.0029 & 47050 \\
$\left(\begin{array}{c}\text { 互相关系数 }>0.85) \\
\text { 793个地震事件 }\end{array}\right.$ & 走时数目 & 33875 & 0.91 & 0.94 \\
\hline
\end{tabular}

a) 考虑到计算内存, 对纵波, 选择初至到时拾取数目 $>40$, 互相关系数 $>0.85$ 的相对走时残差数据; 对横波, 选择初至到时拾取数目 $>10$, 互 相关系数 $>0.85$ 的相对走时残差数据; 互相关系数和均方根误差均为平均值

层追踪地震射线, 计算地壳校正量, 校正相对更为精 确; (2) 在反演矩阵中引入台站效应、事件效应变量 同时反演. 台站效应变量在反演中会吸收台站下方浅 部区域引起的到时偏差(Hung等，2004), 而事件效应 变量则吸收不同事件相对走时数据体间的基线偏移 (Allen等, 2002). 对比经过和未经过地壳校正的成像结 果(网络版附图4和图5), 可以看出地壳“拖尾效应”对最 终的速度模型的影响主要局限在 $60 \mathrm{~km}$ 深度内, 对更深 部的影响很小.

\section{3 成像结果}

本文的结果以横切片(图4和5)和纵切片(图6)的方 式展示，显示的是相对于IASP91地球模型的速度扰动 值. 对比图4 6, 纵、横波成像速度模型在整体上显示 出很好的一致性，但也存在局部的差异. 网络版附图6 和 7 是纵、横波穿过每个网格节点的射线数目分布图. 从图中可以看出，纵、横波主要研究区域网格节点的 射线数目均 $>1500$ (射线密度 $\geq 102$ 条 $/ 100 \mathrm{~km}^{3}$ ), 但纵波 明显地较横波的射线分布更广, 射线密度更大(网络版 附图6a h 和 $a^{\prime} \sim h^{\prime} 、 7 a \sim e$ 和 $\left.a^{\prime} \sim e^{\prime}\right)$. 一方面是由于纵波的 信噪比较横波的更好, 可以拾取更多的源台对走时数 据; 另一方面纵波除了利用与横波相同的 $0.02 \sim 0.1$ 、 $0.1 \sim 0.8 \mathrm{~Hz}$ 频段的走时数据外, 还增加了 $0.8 \sim 2.0 \mathrm{~Hz}$ 高频 段的走时数据. 纵、横波射线空间采样的差异, 以及具 有更窄菲涅尔带的高频纵波走时数据的使用，是纵、 横波成像速度模型存在局部差异的原因. 对比于横波, 纵波的成像速度模型具有更高的分辨率(4.1节), 能够 刻画更加精细的速度结构特征.

在远震体波走时层析成像中, 由于地震射线在地
壳深度内的近垂直入射，射线交叉较少，分辨率不足， 因此本文只讨论 $60 \mathrm{~km}$ 深度以下的速度结构特征. 相比 于前人的体波成像速度模型(较大研究区域范围的工 作, 例如, Huang和Zhao, 2006; Li等, 2006, 2008; Feng 等, 2010; Li和van der Hilst, 2010; Obrebski等, 2012; Wei等, 2012; Zhao等, 2012; Chen等, 2015; Zhao D P等, 2017. 相似研究区域范围的工作, 例如, Zhao等, 2009; Tian等, 2009; Tian和Zhao, 2011; Lei，2012), 本文的 纵、横波速度模型最显著的成像特征是: 在60 250km 深度范围内, 分布在华北克拉通东部南、北缘的高速 异常体H2'和H2"被四周的造山带和郯庐断裂下方的 低速异常体环绕, 低速异常体的形态与分布表现出很 好的构造相关性(图4a e 和 $5 \mathrm{a} \sim \mathrm{e}$ ). 在此章节, 本文的结 果将主要和Tian等(2009)、Lei(2012)的体波速度模型 作对比, 这是因为这两个模型与本文的模型有相似的 研究区域范围和近似的分辨率尺度.

\section{1 鄂尔多斯区域上地幔速度结构}

与前文提及的所有速度模型类似, 鄂尔多斯块体 的下方可以观测到一个显著的高纵、横波速度异常体 $\mathrm{H} 1$, 向下延伸至 300km左右(图4a f、5a f和 $6 \mathrm{a} \sim \mathrm{e} 、 \mathrm{a}^{\prime}$ $\sim \mathrm{e}^{\prime} 、 h \sim i$ 和 $\left.h^{\prime} \sim i^{\prime}\right)$. 得益于更加密集的中西部台阵的覆 盖 (图 1中红色圆圈和红色三角所示), 与 Tian等 (2009)、Lei(2012)的模型相比, 本文的模型勾勒出更 加清晰的鄂尔多斯块体的轮廓(图4a f、5a f、6a e、 $h \sim i 、 a^{\prime} \sim e^{\prime}$ 和 $\left.h^{\prime} \sim i^{\prime}\right)$. 高速异常体H1 随着纬度的降低向下 延伸的深度从北往南逐渐变深(图6a e $、 a^{\prime} \sim \mathrm{e}^{\prime} 、 \mathrm{i}^{\prime}$ 和 $^{\prime}$ ), 局部向下延伸到 $\sim 400 \mathrm{~km}$ (图6c $\sim \mathrm{d} 、 \mathrm{c}^{\prime} \sim \mathrm{d}^{\prime} 、 \mathrm{i}$ 和 $\mathrm{i}^{\prime}$ ), $\mathrm{Li}$ 和 van der Hilst(2010)、Obrebski等(2012)的模型中也观 测到这个往南倾斜的现象. 阿拉善块体下方表现为高 

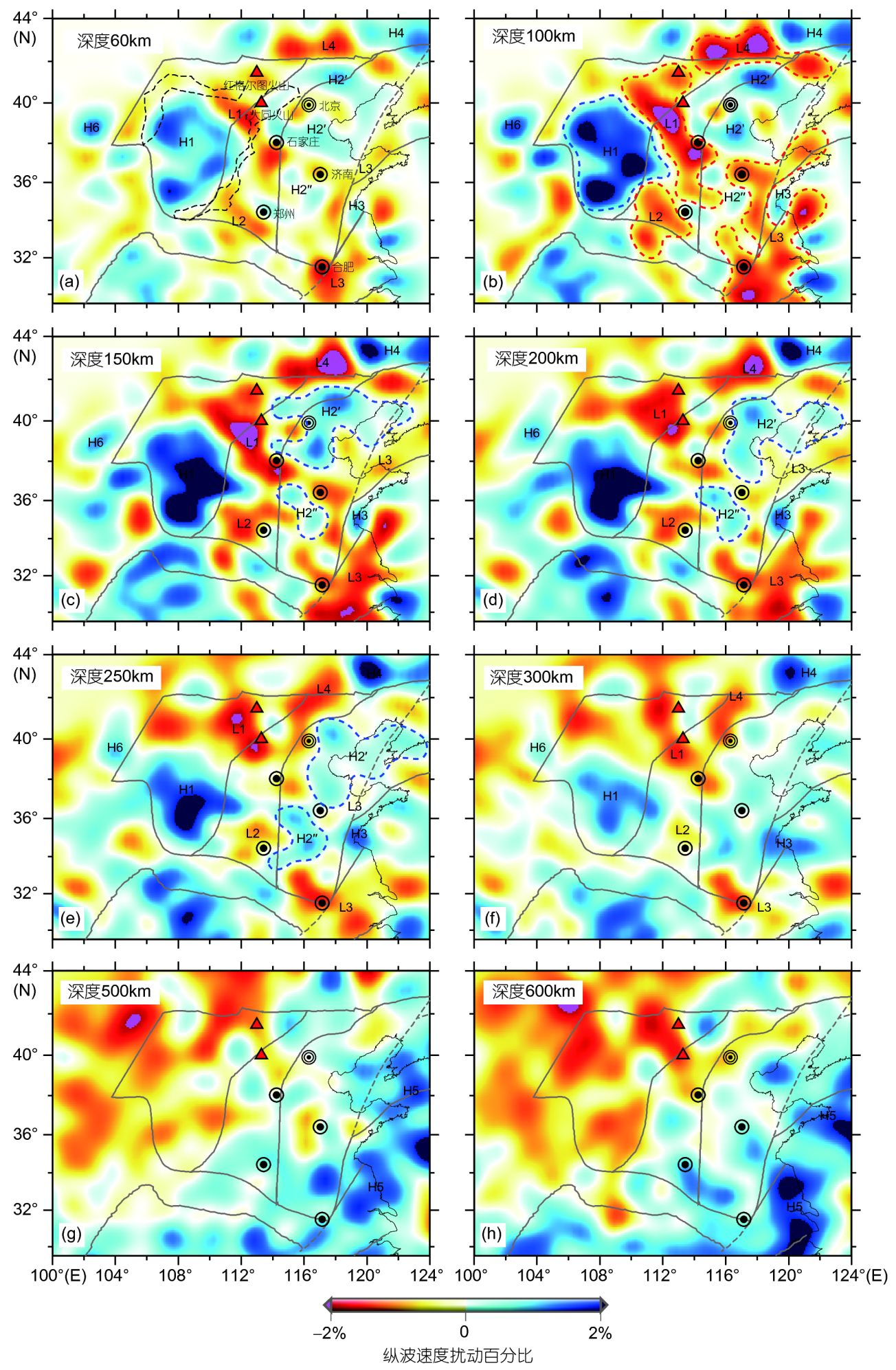

图 4 不同深度的纵波速度扰动模型切片图

红色和蓝色分别表示低纵波速度异常和高纵波速度异常, 异常的幅值见下方的色标; 灰色实线表示构造单元界限, 灰色虚线表示郯庐断裂, (a) 中环绕鄂尔多斯的黑色虚线表示新生代裂谷系, 红色的三角形代表大同火山和红格尔图火山; L1 L4表示不同的低纵波速度异常单元, H1 H5 表示不同的高纵波速度异常单元. (b) (e)中虚线是为了地质解释勾勒出的纵波速度异常体的轮廓 

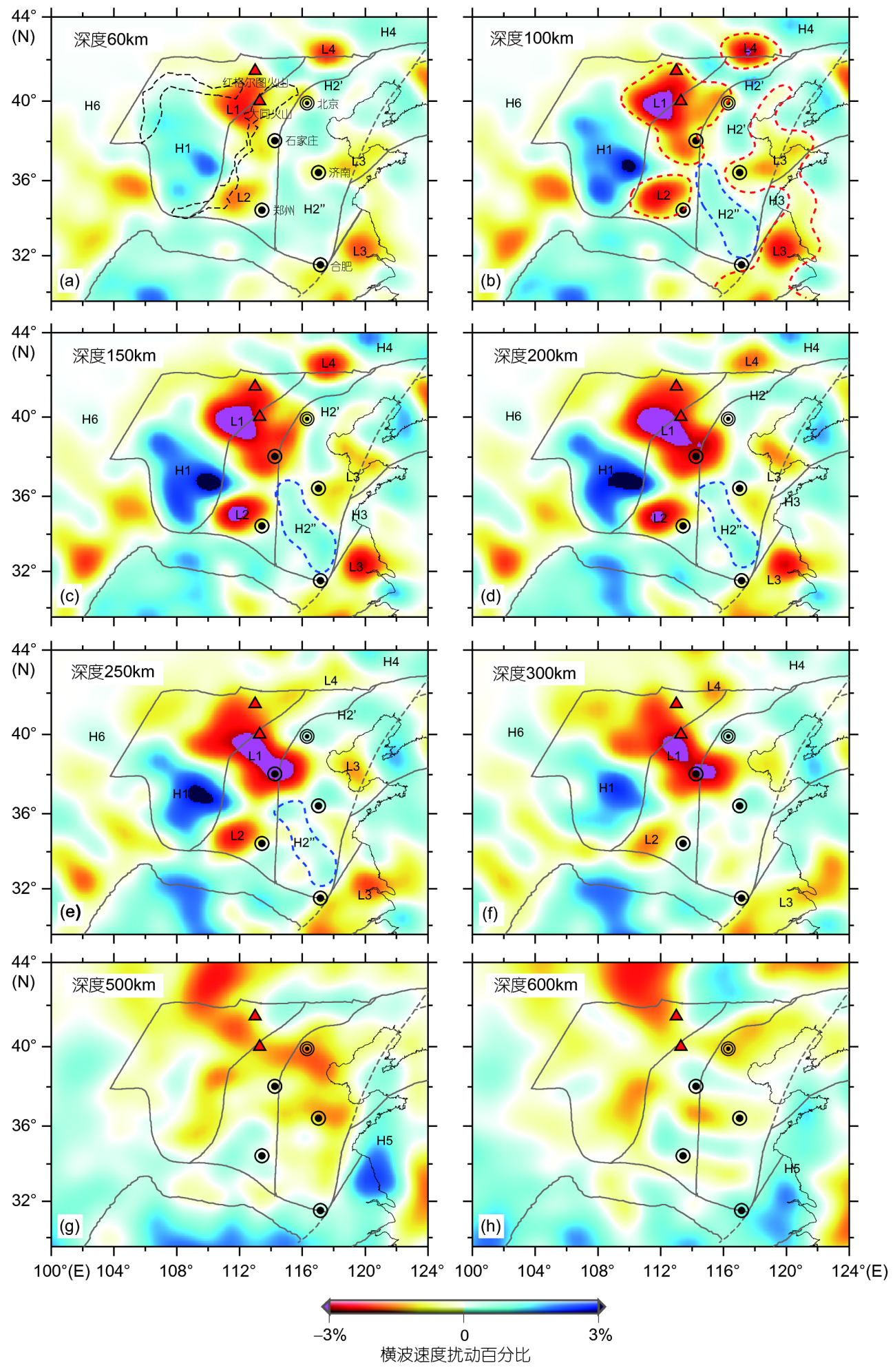

图 5 不同深度的横波速度扰动模型切片图

红色和蓝色分别表示低横波速度异常和高横波速度异常, 异常的幅值见下方的色标; 灰色实线表示构造单元界限, 灰色虚线表示鄰庐断裂, (a) 中环绕鄂尔多斯的黑色虚线表示新生代裂谷系, 红色的三角形代表大同火山和红格尔图火山; L1 L4表示不同的低横波速度异常单元, H1 H5 表示不同的高横波速度异常单元. (b) (e)中虚线是为了地质解释勾勒出的横波速度异常体的轮廓 

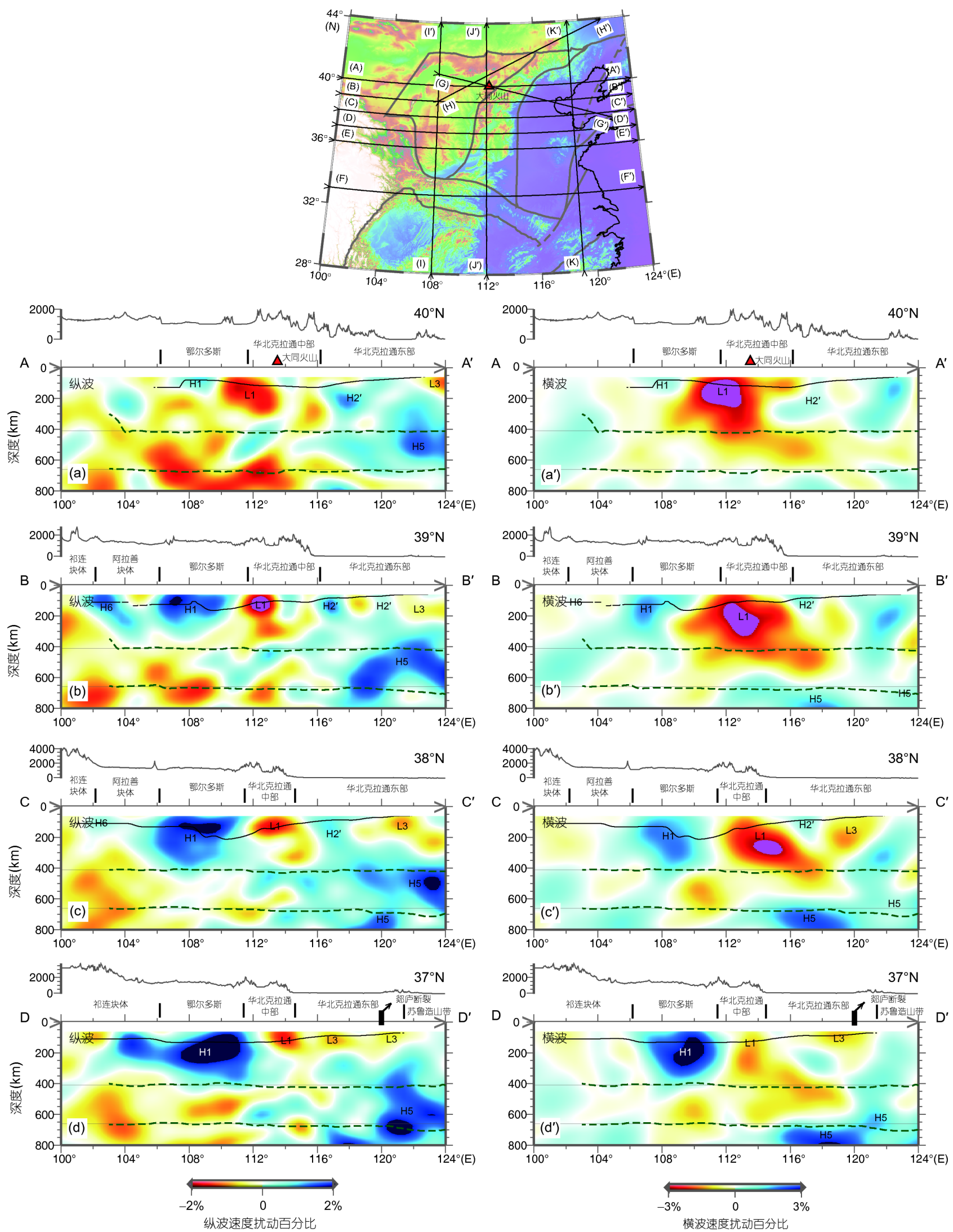

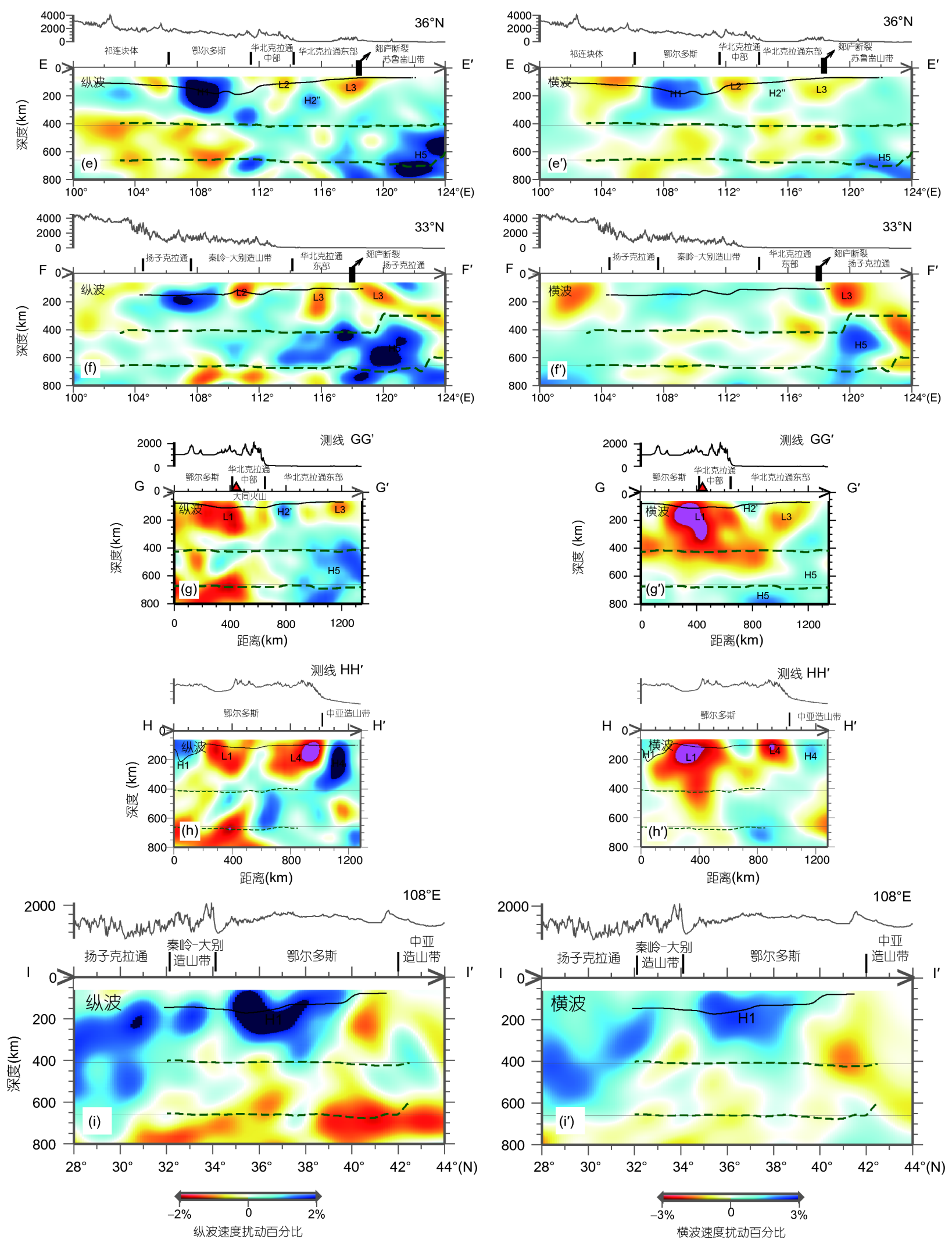

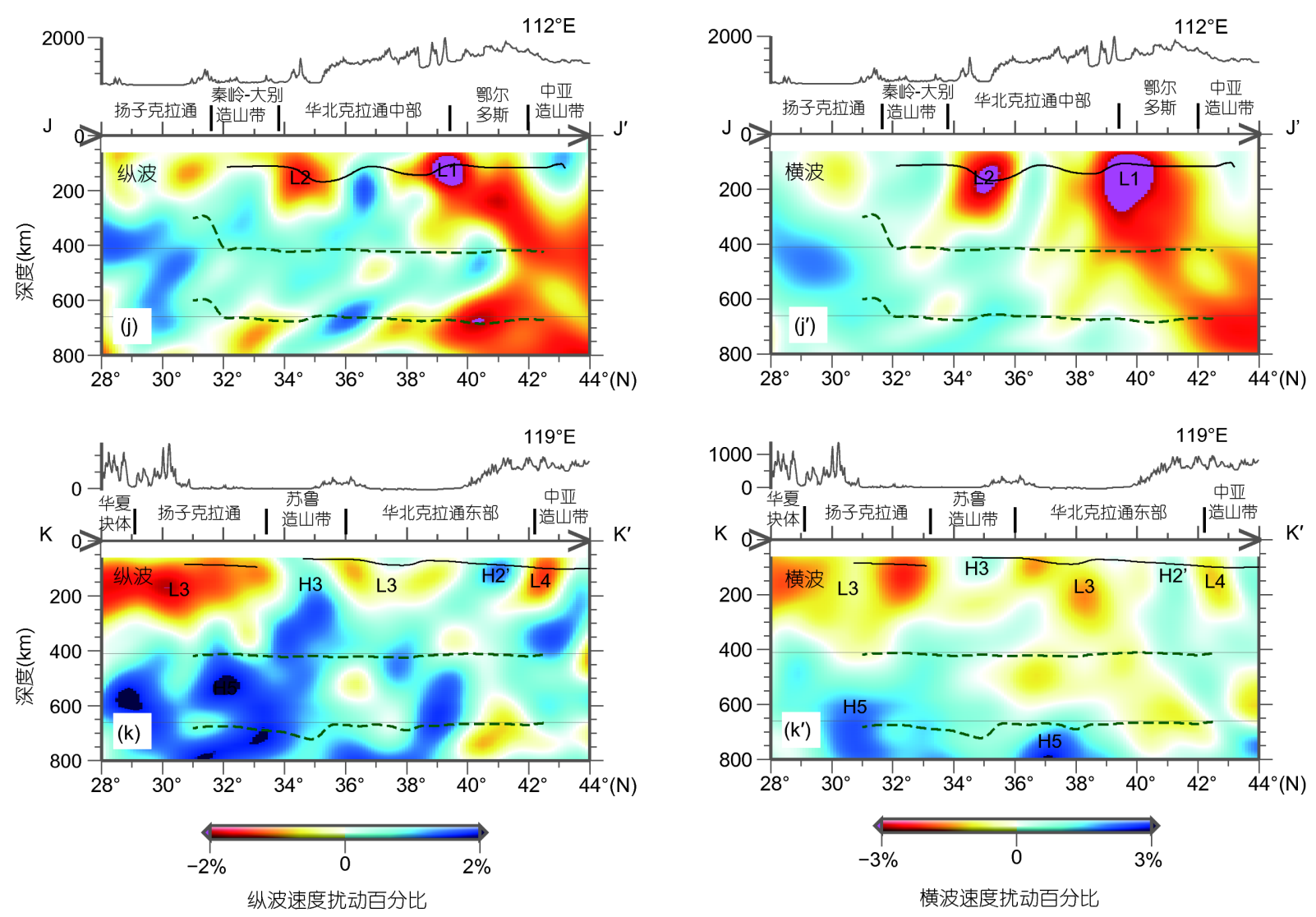

图 6 不同位置的纵、横波速度扰动模型切片图

红色和蓝色分别表示低纵、横波速度异常和高纵、横波速度异常，异常的幅值见下方的色标; 两条灰色实线分别表示理论的410和660km间 断面; 绿色虚线表示接收函数得到的 410 和 $660 \mathrm{~km}$ 间断面(郑天愉等, 2017), 黑色实线表示接收函数得到的岩石圈-软流圈界面(郑天愉等, 2017); L1 L4表示不同的低纵、横波速度异常单元, H1 H5 表示不同的高纵、横波速度异常单元

速异常体H6，而环鄂尔多斯西北缘分隔阿拉善块体与 鄂尔多斯块体的银川-河套裂谷下方则表现为低纵、 横波速度异常(图4a f、5a f、6b、c 和 $\left.\mathrm{b}^{\prime}\right)$. 这个观测结 果与Tian等(2009)的工作一致，但又有些差异. Tian等 (2009)的结果显示阿拉善块体的高速异常分布主要局 限在地壳深度 $(<30 \mathrm{~km})$, 本文的结果则可看出高速异常 体H6从 $60 \mathrm{~km}$ 向下延伸到 $300 \mathrm{~km}$ (图4a f、5a f、6b、 $c$ 和 $b^{\prime}$ ). 而Lei(2012)的结果只在河套裂谷下方观测到低 速异常, 银川裂谷下方则表现为高速异常. 在鄂尔多斯 块体南缘，与扬子克拉通碰撞形成的秦岭造山带下方 $200 \mathrm{~km}$ 深度内, Lei(2012)的模型显示为很强的低速异 常, Tian等(2009)的模型则显示为高速异常, 高速异常 的北部边界甚至进入鄂尔多斯区域. 与这两个模型相 比, 本文的模型可以较为清晰地辨别出鄂尔多斯块体 和扬子块体的边界位置, 扬子块体的北缘似乎比地表
的构造线往北延伸的更远(例如, Li和van der Hilst, 2010; Zhao D P等, 2017), 位于两者之间的秦岭造山 带在上地幔深度内表现为低速异常, 或者强度低于两 者的弱高速异常(图4a f、5a f、6i和i'; Obrebski等, 2012).

\section{2 华北克拉通中部上地幔速度结构}

前人的工作揭示华北克拉通中部的下方为低速异 常, 向下延伸到 400km左右 (例如, Huang 和Zhao, 2006; Li等, 2006; Tian等, 2009; Zhao等, 2009, 2012; Li和van der Hilst, 2010; Lei, 2012; Obrebski等, 2012; Chen等, 2015; Zhao D P等, 2017). Tian等(2009)的模型 显示该区域的低速异常呈NE-SW向，而本文的模型与 Lei(2012)的模型相似, 低速异常的形态表现为弧状(图 $4 \mathrm{a} \sim \mathrm{f}$ 和 $5 \mathrm{a} \sim \mathrm{f})$. 此外, 从本文的模型能进一步看出, 低速 
异常可以分为北部的低速异常体L1和南部的低速异 常体L2两个部分(图4a f、5a f、6j和 $\mathrm{j}^{\prime}$ )，这与Jiang等 (2013)的面波成像的观测结果一致. 北部的低速异常 体L1向北、向南伸展，呈NW-SE走向，向下延伸到地 幔转换带深度(图6a d $\quad g 、 h 、 j$ 和 $a^{\prime} \sim d^{\prime} g^{\prime} \sim h^{\prime} 、 j^{\prime}$ ). 位 于南部的低速异常体L2则表现出与北部的低速异常 体L1明显的空间差异：其分布的范围较小，向下延伸 最深仅至 300km(图4a f、5a f、6e f、j、e $e^{\prime} 、 j^{\prime}$ ). 低 速异常体L1、L2不仅存在空间形态的差异，L1局部地 区呈现更强的异常幅值，对于纵、横波异常强度分别 $>2 \%$ (纵波速度扰动)和 $>3 \%$ (横波速度扰动)(图 $4 \mathrm{a} \sim \mathrm{f}$ 、 $5 a \sim f 、 6 a \sim c 、 j$ 和 $\left.a^{\prime} \sim c^{\prime} 、 g^{\prime} 、 h^{\prime} 、 j^{\prime}\right)$, 该高异常幅值区域 空间上对应于地表的大同火山(图4a f、5a f、6a、g 和 $\left.a^{\prime} 、 g^{\prime}\right)$. 在两个低速异常体L1、L2之间，临近山西陕西裂谷的转换拉伸区域，本文的模型观测到一个从 $60 \mathrm{~km}$ 延续到 $300 \mathrm{~km}$ 深度的高速异常，横向上表现为与 西侧的鄂尔多斯块体相连(图4a f、5a f、6j和 $\left.\mathrm{j}^{\prime}\right)$. Tian 等(2009)、Lei(2012)的体波模型也有类似的现象，而 Jiang等(2013)的面波成像的模型则显示该高速异常只 延伸到 100km，被狭窄的低速异常带分隔成一个独立 于鄂尔多斯块体的区域.

\section{3 华北克拉通东部上地幔速度结构}

与鄂尔多斯块体和华北克拉通中部地区不同，华 北克拉通东部的上地幔表现出非均匀的小尺度横向速 度结构特征. 相对于前人的模型, 本文的模型观测到新 的速度结构特征: 华北克拉通的北缘, 中亚造山带下方 $300 \mathrm{~km}$ 深度内呈现为明显的低纵、横波速度异常 L4, L4的走向从 $200 \mathrm{~km}$ 深度内的E-W向转为 $200 \sim 300 \mathrm{~km}$ 深 度内的NE-SW向(图4a f、5a f和 $\left.6 h^{\prime} 、 h^{\prime}\right)$. 在低速异常 体L4的北侧，燕山块体和松辽盆地之间的下方存在一 个往西南方向倾斜的高纵、横波速度异常体 $\mathrm{H} 4$ ，向下 延伸到 $350 \mathrm{~km}$ 左右(图4a f、5a f、6h和 $\mathrm{h}^{\prime}$ ). Lebedev和 Nolet(2003)、Li等(2006)、Tian等(2009)的模型在该区 域也观测到相同的结果. 高速异常体H4可能是往西延 伸的松辽盆地下方的元古代岩层(Zhang M等，1998), 低速异常体 L4在 200 300 km深度的走向可能与此 相关.

位于华北克拉通东部的东侧，阯庐断裂的下方是 显著的低纵、横波速度异常体L3，这与前人的观测结 果一致(例如, Huang和Zhao, 2006; Tian等, 2009; Zhao
等, 2009, 2012; Lei, 2012; Obrebski等, 2012), 但不同模 型之间低速异常体的成像深度存在着差异. Tian等 (2009)的模型显示䣊庐断裂下方的低速异常延伸到 400km深度；Zhao等(2012)的模型观测到的深度更深， 到达地幔转换带内部; 而本文的模型则揭示低速异常 体L3 只存在于 $200 \sim 300 \mathrm{~km}$ 深度之上(图4a f、5a f、 6a $\sim \mathrm{g} 、 \mathrm{k}$ 和 $c^{\prime} \sim \mathrm{g}^{\prime} 、 \mathrm{k}^{\prime}$ ), 这与Huang和Zhao(2006)、Lei (2012)、Obrebski等(2012)的模型一致. Lei(2012)的模 型显示低速异常体L3、L1在200km深度汇合向下延伸 到下地幔, 形成Y型低速异常形态. 但从与Lei(2012)相 同的剖面位置(图6g和 $g^{\prime}$ )可以看出, 本文模型中的低速 异常体L1、L3被高速异常体H2'隔断，下方是高速异 常体H5, 并不存在Y型的低速异常形态.

在低速异常体L1、L2、L3和L4环绕的中间, 主要 位于华北克拉通东部的燕山地区以及南缘地区(石家 庄、济南、合肥和郑州包围的区域)的下方 $250 \mathrm{~km}$ 深 度内可以观测到高纵、横波速度异常体 $\mathrm{H} 2$ '和H2"(图 4a e 、5a e 、6a c 、 $、 g 、 k$ 和 $\left.a^{\prime} \sim c^{\prime} 、 e^{\prime} 、 g^{\prime} 、 k^{\prime}\right)$. 前人 的模型(Huang和Zhao, 2006; Tian等, 2009; Zhao等, 2009，2012; Li和van der Hilst，2010; Lei，2012; Obrebski等，2012)也显示在华北克拉通东部的下方存 在着高速异常, 但不同的模型存在着一些差异. 在Tian 等(2009)的模型中，该高速异常体呈N-S向，从 $80 \mathrm{~km}$ 延 伸到300km深度; 在Lei(2012)的模型中, 则表现为弧状, 从浅部延伸到 $200 \mathrm{~km}$ 深度; 而在Huang和Zhao(2006)、 Li和van der Hilst(2010)、Obrebski等(2012)的模型中, 该高速异常则分别分布在 $300 \sim 400 \mathrm{~km}$ 深度范围、 150 350km深度范围和 $100 \mathrm{~km}$ 深度范围内. 本文模型 中的高速异常体 $\mathrm{H} 2$ '和 $\mathrm{H} 2^{\prime \prime}$ 的形态较为复杂、异常幅 值不高, 表现为 $1 \%$ 幅值的纵波速度扰动和 $1.5 \%$ 幅值 的横波速度扰动，而位于燕山块体区域的异常幅值相 对较高. 相比于前人的模型，本文的模型也显示了更 小尺度的结构特征，苏鲁造山带的下方表现为高纵、 横波速度异常H3(图4a f、5a f 和 $6 \mathrm{k} 、 \mathrm{k}^{\prime}$ ). 由于在该区 域纵波具有比横波更密集的射线覆盖(网络版附图6和 7), 纵波的速度结构显得相对清晰: H3的北侧与烟台青岛-五莲断裂吻合，西侧与矨庐断裂吻合，表现出很 好的构造相关性(图4a f 和图 $5 \mathrm{a} \sim \mathrm{f}$ ).

\section{4 地幔转换带内速度结构}

华北克拉通东部下方的地幔转换带内存在显著的 
高纵、横波速度异常体H5(图4g、h, 5g、h, 6a g, k和 $\mathrm{b}^{\prime} \sim \mathrm{g}^{\prime} 、 \mathrm{k}^{\prime}$ ), 这与前人模型的观测结果一致(例如, Lebedev和Nolet, 2003; Huang和Zhao, 2006; Li等, 2006, 2008; Tian等, 2009; Feng等, 2010; Li和van der Hilst, 2010; Lei, 2012; Obrebski等, 2012; Zhao等, 2012; Chen 等, 2015; Zhao D P等, 2017), 高速异常体H5可能代表 了俯冲停滞的太平洋板片前沿. 不同模型之间地幔转 换带内高速异常体的形态存在着差异：Lei(2012)模型 中的高速异常体西侧位于 $112^{\circ} \mathrm{E}$ ，且在大同火山区域 下方存在一个缺口; 而Huang和Zhao(2006)、Tian等 (2009)、Li和van der Hilst(2010)模型中的高速异常体 则平躺在地幔转换带内, 最西端位于 $119^{\circ} \sim 120^{\circ} \mathrm{E}$. 本 文的纵、横波速度模型在地幔转换带深度具有很好的 射线覆盖(网络版附图 $6 \mathrm{~g} \sim \mathrm{h} 、 6 \mathrm{~g}^{\prime} \sim \mathrm{h}^{\prime}$ 和 7) 和分辨率 ( 168km, 网络版附图8 11, 4.1节). 从横切片图(图4g、 $\mathrm{h}$ 和 $5 \mathrm{~g} 、 \mathrm{~h})$ 可以看出, 地幔转换带内高速异常体 $\mathrm{H} 5$ 的最 西端大概位于炻庐断裂的下方, $\sim 118^{\circ} \mathrm{E}$. 从纵切片图 (图6a $\sim \mathrm{g} 、 \mathrm{k}$ 和 $\mathrm{b}^{\prime} \sim \mathrm{g}^{\prime} 、 \mathrm{k}^{\prime}$ ) 可以看出, 高速异常体H5较前 人的模型(例如, Huang和Zhao, 2006; Tian等, 2009; Li 和van der Hilst, 2010; Lei, 2012)表现出非均一的形态 特征: H5 不是完全地平躺在地幔转换带内, 部分上趐 至410km间断面之上(图6a d d f、g、f'; Wei等, 2012), 部分发生弯曲变形(图6b、f、 $\mathrm{f}^{\prime}$ ), 部分穿过了 660km间 断面下沉至下地幔(图6c $\sim \mathrm{g} 、 \mathrm{k}$ 和 $b^{\prime} \sim \mathrm{g}^{\prime} 、 \mathrm{k}^{\prime}$ ), 下沉部分 的最西端位于 $\sim 116^{\circ} \mathrm{E}$ (图6b f 和 $\left.b^{\prime} \sim f^{\prime}\right)$. 在高速异常体 $\mathrm{H} 5$ 存在的区域, 地幔转换带的厚度也发生了明显地增 厚(Chen和Ai，2009; Xu等, 2011; 郑天愉等, 2017; 图 $6 a \sim g 、 a^{\prime} \sim g^{\prime} 、 k$ 和 $k^{\prime}$ 中绿色虚线所示).

\section{4 理论测试}

本文进行了详细的理论测试以验证成像模型中主 要异常特征的可靠性. 理论测试的步骤如下: 在模型空 间内给定理论的速度模型, 采用与实际地震数据反演 中相同的台站、地震位置和射线路径计算给定速度模 型的理论到时, 运用与实际地震数据反演相同的反演 方法和参数对理论到时反演成像. 通过对比理论输入 模型和反演输出模型，可以评估模型空间内的分辨率 优劣情况. 本文进行了两种类型的理论测试: 检测板 分辨率测试和速度异常体形态的恢复测试.

\section{1 检测板分辨率测试}

检测板分辨率测试用于检测地震数据的分辨尺度 和评估研究区域的射线覆盖情况. 通过给定不同网格 距大小的输入模型，本文进行了许多检测板分辨率测 试, 输入模型的尺度为4倍网格距(经、纬度和深度向 的尺度分别为 $1.5^{\circ} 、 1.25^{\circ}$ 和 $91 \mathrm{~km}$, 即 $\sim 134 \mathrm{~km} \times$ $138 \mathrm{~km} \times 91 \mathrm{~km}), 5$ 倍网格距(经、纬度和深度向尺度为 $1.88^{\circ} 、 1.56^{\circ}$ 和 $114 \mathrm{~km}$, 即 $\sim 168 \mathrm{~km} \times 173 \mathrm{~km} \times 114 \mathrm{~km}$ ) 和 6 倍网格距 (经、纬度和深度向尺度为 $2.25^{\circ} 、 1.88^{\circ}$ 和 $137 \mathrm{~km}$, 即 $202 \mathrm{~km} \times 208 \mathrm{~km} \times 137 \mathrm{~km}$ ). 对于纵、横波, 分 别在模型空间的网格点上交替给定 $2.0 \%$ 和 $3.0 \%$ 幅值的 正、负纵、横波速度扰动. 在计算得到的理论到时中 加入 $10 \%$ 的高斯随机噪声以模拟实际走时数据的数据 误差. 网络版附图8 11是采用与实际地震数据反演相 同的算法(LSQR，最小二乘正交分解法)和参数 (纵、横 波的阻尼因子分别为 40.0 和 50.0 , 卷积平滑因子的互相 关长度均为 2 倍网格距)得到的检测板测试结果模型.

网络版附图 8 是 4 倍网格距的检测板测试输出模 型. 模型显示纵波在30 300km(网络版附图8 a c c)、横 波在30 150km(网络版附图8d 8f) 深度内成像的速度 异常幅值虽然有所丢失, 但形状却可以很好的恢复. 这 表明纵、横波分别在30 300和30 150km深度区间具 有很好的空间分辨率, 分辨率尺寸 $134 \mathrm{~km}$. 对于 5 倍网 格距的输出模型(分辨尺寸 $168 \mathrm{~km}$ ), 纵波(网络版附图 9a e 和10a e) 和横波(网络版附图9f j 和附图 10f j ) 分 别在30 800和30 700 $\mathrm{km}$ 深度内显示出很好的分辨率. 从横波的纬度切片(网络版附图10f j j) 可以看出, 横波 在30 250、400 700 km深度内表现出较250 400 km深 度范围更好的成像结果，这可能是横波射线密度分布 不均的结果(网络版附图 $6 \mathrm{a}^{\prime} \sim \mathrm{h}^{\prime}$ ). 在网络版附图11(6倍 网格距, 分辨率 $202 \mathrm{~km}$ ) 的输出模型中, 纵、横波在整 个研究区域范围内均表现出很好的空间分辨率. 总之, 检测板分辨率测试结果显示: 纵波在30 300 $\mathrm{km}$ 深度内 分辨率尺寸 $134 \mathrm{~km}$ ，在300 800km深度内分辨率尺寸 $\sim 168 \mathrm{~km}$; 横波的分辨率尺寸从 $30 \sim 150 \mathrm{~km}$ 深度内的 $\sim 134 \mathrm{~km}$ 变成150 700km深度内的 $168 \mathrm{~km}$, 至更深处 $(>700 \mathrm{~km}$ 深度)的 $202 \mathrm{~km}$.

\section{2 恢复测试}

恢复测试用于检测给定输入模型的形态重建结果 
的准确性以及“拖尾效应”的影响程度. 通过改变输入 的速度异常模型的直径和形态，本文实施了许多恢复 测试，恢复测试的步骤与检测板分辨率测试类似．根 据成像速度模型(图4 6)的异常体特征，本文在不同深 度区间设定了不同直径、不同倾角的高、低速异常体 (网络版附图12和13中灰色虚线所示), 对于纵、横波, 给定模型的速度扰动分别为 $\pm 2 \%$ 幅值的纵波速度扰动 和 $\pm 3 \%$ 幅值的横波速度扰动. 恢复测试的输出模型显 示，速度异常体能很好地恢复其异常幅值和形态，横 向上的分辨率明显优于垂向上的分辨率(网络版附图 $12 c \sim d 、 g \sim h$ 和 $13 b 、 d 、 f 、 h 、 i \sim j) ，$ 这是由远震体波层 析成像的性质决定的. 不同的测试显示, 异常体底界面 深度不同, “拖尾效应”的影响不同. 对于异常体底界面 位于 $200 \mathrm{~km}$ 深度时，垂向的“拖尾效应”十分微弱，可以 忽略不计(网络版附图13b、d中H5, H5 宽200km，深 $200 \mathrm{~km}$ ). 而异常体底界面位于 $280 \mathrm{~km}$ 时，垂向的“拖尾 效应”变得较为明显, $70 \mathrm{~km}$ (网络版附图 $12 \mathrm{c} 、 \mathrm{~g}$ 中 $\mathrm{H} 1$, $\mathrm{H} 1$ 宽 $400 \mathrm{~km}$ ，深 $280 \mathrm{~km})$. 无论是垂直形态，或倾斜形 态，异常体底界面位于 $410 \mathrm{~km}$ 时，垂向的“拖尾效应”为 $\sim 40 \mathrm{~km}$ (网络版附图12c、g中 $\mathrm{g} 1$; 网络版附图13f、h中 L2; L1、L2宽 $400 \mathrm{~km}$, 深 $410 \mathrm{~km})$. 但当地幔转换带和上 地幔同时存在高速异常体时，垂向的“拖尾效应”变得 特别明显(网络版附图12d和h). 因此本文模型中高速 异常体从上地幔贯穿到地幔转换带的现象，可能反映 了上地幔和地幔转换带同时存在着高速异常体. 本文 也测试了地幔转换带和下地幔同时存在高速异常体的 恢复情况. 输出模型显示地幔转换带内的高速异常体 (网络版附图 $13 \mathrm{i} 、 \mathrm{j}$ 中H6、H6 宽 $120^{\circ} \sim 124^{\circ} \mathrm{E}$, 深 410 600 km) 和下地幔内的高速异常体(网络版附图 $13 \mathrm{i} 、 \mathrm{j}$ 中 H7, H7宽 $115.6^{\circ} \sim 120.6^{\circ} \mathrm{E}$ ，深700 800km) 可 以很好的分辨出来, 由于大量射线来自东部且异常体 位于研究区域的东缘(网络版附图7), 恢复出的高速异 常体存在着一定的“拖尾效应”(附图13i和13j). 对比 纵、横波输出模型的异常幅值可以看出, 纵波输出模 型的异常幅值损失 $25 \%$, 而横波则损失 $~ 57 \%$, 这可能 是纵、横波地幔转换带内高速异常体成像差异的 原因.

\section{5 讨论}

利用零均值相对走时残差数据的成像方法得到的
是相对速度扰动值, 主要探讨异常体间的速度差异 (Wawerzinek等, 2013). 地震层析成像横向非均匀性起 因的研究对地球内部过程有重要的动力学指示作用 (Rocha等, 2011). 温度和物质组分是影响纵、横波速 度异常最重要的两个因素，分别代表了不同的动力学 意义, 但是仅从成像的结果很难区分两者的作用(Karato和Karki, 2001). 基于岩石物理实验和相应的正演计 算(Goes等, 2000; Cammarano等, 2003; Hieronymus等, 2007; Zhang等, 2016), 温度是影响上地幔速度异常最 主要的因素. 在 $400 \mathrm{~km}$ 深度内物质组分相对于温度的 影响是次要的, 但随着深度的增加物质组分的重要性 变高; 在干地幔假设下, $\pm 1 \%$ 幅值的纵波速度扰动大 约对应于温度100 200K的变化(Cammarano等，2003). 在没有强烈亏损、富镁方辉橄榄岩的情况下, 速度异 常受物质组分的影响可以忽略( $<1 \%$ 的速度扰动)(Sobolev等, 1995).

在活动的构造区域, 除了温度, 部分熔融和流体也 会影响地震波参数(Anderson和Sammis，1970; Artemieva等, 2004). 升温和流体加入均可引起部分熔融, 正演计算表明，上地幔内每百分比的部分熔融分别会 引起至少 3.6\%的纵波速度降和7.9\%横波速度降(Hammond和Humphreys, 2000).

对比岩石圈-软流圈界面深度和成像的结果可以 看出，华北克拉通东部减薄的岩石圈下方存在明显的 低速异常区域(图6a g、j和 $a^{\prime} \sim g^{\prime} 、 j^{\prime}$ ). 接收函数在该区 域的下方观测到显著的、尖锐的岩石圈-软流圈转换 带, 表明这些低速异常不仅是温度作用的结果, 还伴随 着挥发分(气体和流体)或部分熔融的作用(Rychert等, 2005; Chen, 2010). 因此本文模型中的速度异常体可能 反映了温度和流体共同作用的结果.

\section{1 华北克拉通显著的横向不均匀性}

层析成像的结果显示，纵、横波速度模型在上地 幔表现出的横向不均匀性与构造单元的空间位置、构 造活化和岩浆事件的分布以及区域动力学事件有很好 的对应关系. 本文的模型和前人体波成像模型(例如, Huang和Zhao, 2006; Li等, 2006, 2008; Sun等, 2008a, 2008b; Tian等, 2009; Zhao等, 2009, 2012; Li和 van der Hilst, 2010; Feng等, 2010; Lei, 2012; Obrebski等, 2012; Chen等，2015)的整体特征一致，表明了本文成像结果 的可靠性, 同时进一步揭示了一些新的构造特征. 新的 
模型为探究华北克拉通岩石圈改造、减薄的动力学过 程和空间尺度提供了重要的深部结构约束.

\subsection{1 华北克拉通中、西部破坏的局限性}

鄂尔多斯块体被北部的内蒙造山带、西部的祁连 造山带、南部的秦岭造山带和东部的中央造山带包 围, 并发育环鄂尔多斯周缘的新生代银川-河套裂谷和 山西-陕西裂谷. 成像的结果显示，鄂尔多斯块体下方 是延伸到 $300 \mathrm{~km}$ 深度左右的高纵、横波速度异常(图 $4 a \sim f 、 5 a \sim f 、 6 a \sim e 、 i$ 和 $\left.a^{\prime} \sim e^{\prime} 、 i^{\prime}\right)$. 由于远震体波成像在 垂向的“拖尾效应”影响，鄂尔多斯块体实际的深度 230km左右(4.2节所述)，与接收函数、面波成像揭示 的岩石圈 160 300km深度 (图6中黑色实线、图7a中 等值线所示；Bao等，2011；Chen等，2014；Jiang等， 2013; 郑天愉等, 2017)相近. 鄂尔多斯块体密度较低 (Li和Yang, 2011), 地表热流值较低( $40 \mathrm{~mW} \mathrm{~m}^{-2}$ )(汪集 旸等, 1996; Hu等, 2000), 没有壳内变形(Shen等, 2000; 王敏等, 2003)，这些特征表明鄂尔多斯块体下方保留 有前寒武纪陆核，在构造事件中保持相对稳定的状态. 与稳定的鄂尔多斯块体不同，华北克拉通中、西部强 烈的岩石圈伸展变形主要集中在新生代裂谷区(Zhang Y Q等, 1998; Zhang等, 2003). 裂谷区下方表现为明显 的低纵、横波速度异常(图4a f 和 $5 \mathrm{a} \sim \mathrm{f}$ ), 下方的岩石圈 和地幔转换带的厚度均发生了减薄, 地幔转换带厚度 $<245 \mathrm{~km}$ (Chen和Ai，2009; 图6a e、6g j、a $\mathrm{a}^{\prime} \sim \mathrm{e}^{\prime} 、 \mathrm{~g}^{\prime} \sim \mathrm{j}^{\prime}$ 中绿色虚线所示), 岩石圈厚度 $<100 \mathrm{~km}$, 且存在着空间 上的变化(Chen, 2010; 图7a中等值线所示). 新生代玄 武岩的地球化学数据表明, 裂谷区玄武岩浆的源区由 老至新逐渐变浅, 暗示新生代时期岩石圈正在不断减 薄(Xu等, 2004; Xu, 2007; 徐义刚等, 2009).

华北克拉通中部的岩石圈发生了一定程度的减 薄, 岩石圈厚度 $100 \mathrm{~km}$ 变化到 $200 \mathrm{~km}$, 最薄处位于山 西-陕西裂谷系北部的伸展区(图7a中等值线所示). 山 西-陕西裂谷系在 $6 \mathrm{Ma}$ 发生活化，代表着新生代岩石 圈减薄和改造的最近一个阶段(王乃梁等, 1996; Zhang等，2003). 华北克拉通中部的岩浆事件主要发 生在晚白严-早第三纪(Tang等，2006；Xu等，2008; Zhao等, 2013), 远早于裂谷活化的时间. 从成像的结 果也可以看出, 低速异常体不局限于裂谷区, 而与浅表 的红格尔图火山、大同火山(图4a 4f和 $5 \mathrm{a} \sim 5 \mathrm{f}$ )和新生 代的岩浆事件(图7b中黄色圆圈所示)有很好的空间对
应关系. 在大同火山的下方并未观测到Lei(2012)模型 中延伸至下地幔的低速异常(图6g和 $g^{\prime}$ ), 本文的模型 显示大同火山下方的低速异常体L1从浅部一直延伸 至地幔转换带的顶部(图6a d $、 g 、 h 、 j$ 和 $a^{\prime} \sim d^{\prime} 、 g^{\prime} \sim h$ '、 j'; 例如, Huang和Zhao, 2006; Li和van der Hilst, 2010; Zhao等, 2012). 相比于纵波, 横波模型中L1向下 延伸的更深，至地幔转换带内部 $550 \mathrm{~km}$ (图6a d $\mathrm{g}$ 、 $h 、 j$ 和 $\left.a^{\prime} \sim d^{\prime} 、 g^{\prime} \sim h^{\prime} 、 j^{\prime}\right)$. 从恢复测试的结果来看 $(4.2$ 节 所述), 不太可能是“拖尾效应”的影响, 可能反映了地 幔转换带内部受俯冲板片、温度和成份改变导致的速 度变化(Schmandt和Humphreys, 2010). 低速异常体L1 在横向上呈现NW-SE的走向 (图 $4 \mathrm{a} \sim \mathrm{f}$ 和 $5 \mathrm{a} \sim \mathrm{f}$ )与 GPS数 据(Wang等，2001)、板片绝对运动方向(Gripp和Gordon，2002)以及各向异性的快轴方向(Huang等，2011; Chang等, 2012)一致. 横波分裂(Huang等, 2011; Zhao 等，2011; Chang等，2012)和纵波径向各向异性成像 (Wang等，2014)观测到L1下方存在着水平向的地幔流 动, 可能造成了 $\mathrm{L} 1$ 的走向分布. 南部的低速异常体L2 延伸到约200 300 km深度(图6e f、j和 $e^{\prime} 、 j^{\prime}$ ), 新生代 岩浆事件较少(图7b中黄色圆圈所示), 保留较厚的岩 石圈约160 200km(图7a中等值线所示), 呈现负的纵波 径向各向异性(垂向的纵波速度大于横向的纵波速度), 与L1存在明显的空间差异性. 华北克拉通中部下方的 地幔转换带厚度发生了减薄(图6中绿色虚线所示; Xu 等, 2011; 郑天愉等, 2017), 延伸至地幔转换带的低速 异常体 L1 可能与俯冲的太平洋板片有关(Huang和 Zhao, 2006; Zhao, 2007; Zhao和Xue, 2010). 临近山 西-陕西裂谷的转换拉伸区域, 在低速异常体L1、L2之 间存在的高速异常体(图4a f和 $5 \mathrm{a} \sim \mathrm{f}$ ) 由于受限于横向 分辨率的尺寸, 则无法判断它是鄂尔多斯块体的一部 分, 或是残留的华北克拉通中部的岩石圈(Jiang等, 2013).

\subsection{2 华北克拉通东部破坏的空间非均匀性}

与鄂尔多斯块体一样, 华北克拉通东部的四周也 环绕着造山带, 北缘是晚二叠-早三叠的中亚造山带, 西缘是元古代的中央造山带, 南缘和东缘是三叠纪碰 撞形成的秦岭-大别-苏鲁造山带. 但不同的是华北克 拉通东部的岩石圈发生了普遍的、显著的减薄, 岩石 圈厚度约 60 100km(图7a中等值线所示; Chen等, 2006, 2008; Chen, 2010; Li等, 2013; 郑天愉等, 2017), 

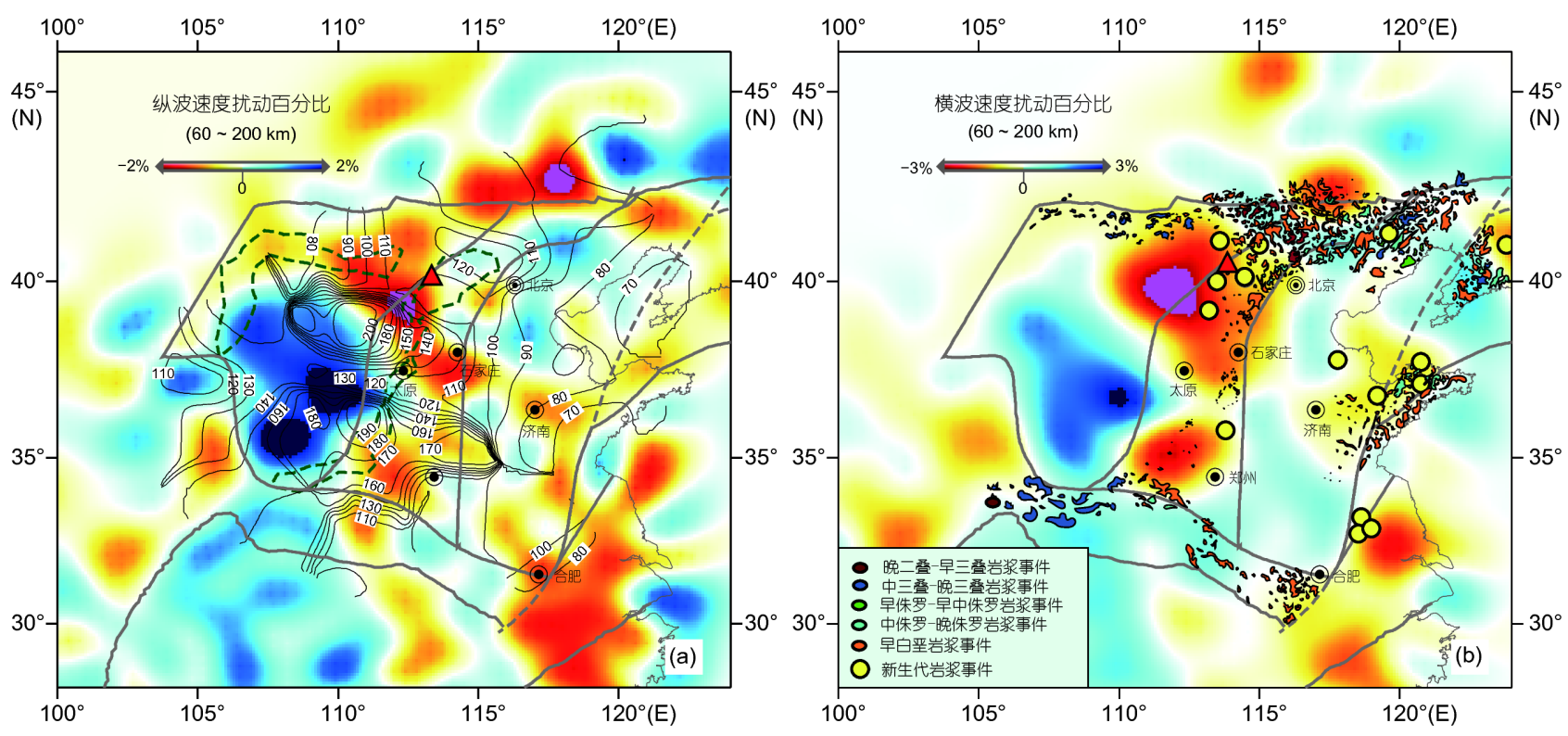

图 7 岩石圈-软流圈界面深度图和不同时代的岩浆事件分布图

(a)和(b)的底图分别为60 200 km深度的纵、横波平均速度扰动图. (a)中岩石圈-软流圈界面深度来自接收函数的结果(郑天愉等, 2017); (b)中 岩浆事件的位置参考吴福元等(2008)、徐义刚等(2009)和Zhang等(2014)

最薄处位于郯庐断裂下方，约60 70km(图7a中等值线 所示; Chen等, 2006; Chen, 2010). 中亚造山带和郯庐 断裂下方是显著的低纵、横波速度异常体L4和L3(图 $4 \mathrm{a} \sim \mathrm{e}$ 和 $5 \mathrm{a} \sim \mathrm{e})$. 地球化学数据显示中亚造山带自晚石炭 纪起经历了多期构造活化(李洪颜等, 2009; 徐义刚等, 2009; Zhang等, 2014; 图7b), 㸚庐断裂则被认为在中生 代-新生代的华北克拉通东部软流圈物质上涌和岩石 圈活化中起到重要作用(例如，Xu，2001; Zheng等, 2008; Chen, 2010). 动力学数值模拟实验显示, 构造薄 弱带(如碰撞造山缝合带和断裂)作为缓冲带可以保护 克拉通免受地幔对流产生的应力影响而保持长期稳定 (Lenardic等, 2000, 2003). 克拉通内部的高粘度表现出 的高屈服应力的动力学机制是克拉通稳定的另一因素 (Lenardic等, 2003; Hieronymus等，2007)，但当克拉通 位于俯冲区域时，这一机制将不能继续维持克拉通的 稳定(Lenardic等，2003). 华北克拉通东、西部地质演 化的不同可能反映了俯冲背景下近场、远场作用的 差异.

华北克拉通东部被低速异常体L1、L2、L3和L4 环绕的区域可以观测高速异常体H2'和H2"(图4a e 、 5a $、 6 a \sim c 、 e 、 g 、 k$ 和 $\left.a^{\prime} \sim c^{\prime} 、 e^{\prime} 、 g^{\prime} 、 k^{\prime}\right)$. 相比于南 缘的H2”, 燕山块体下方的高速异常体 $\mathrm{H} 2$ '异常幅值最
强, 面波成像同样显示该区域为高速异常, 下方的绝对 横波速度约4.45 4.6 $\mathrm{km} \mathrm{s}^{-1}$, 具有典型的克拉通速度特 征(Tang和Chen, 2008). 与华北克拉通东部其他地区呈 现的高热流值(约60 75 $\mathrm{mW} \mathrm{m}^{-2}$ )不同，燕山块体的地 表热流值约30 50mW m${ }^{-2}$ (Hu等，2000，2001; Wang， 2001)，对应于较厚的“热”岩石圈厚度(100 120km)(何 丽娟等，2001)，与地震学方法得到的岩石圈厚度 ( 80 110km, 局部>120km; 图6b)一致(Chen, 2010; 郑 天愉等，2017). 对火成岩的研究发现，燕山地区存在 $<100 \mathrm{Ma}$ 的埃达克质火成岩, 反映了燕山区域可能处于 弱伸展的构造背景(Davis, 2003). 这些特征暗示燕山块 体下方可能存在较厚的岩石圈根，在克拉通破坏过程 中的改造作用不明显. 华北克拉通东部南缘的高速异 常体H2"主要集中在石家庄、郑州、合肥和济南围成 的区域内, Tian等(2009)和Lei(2012)的结果也显示这个 区域为高速异常. 对新生代玄武岩幔源包体的研究, 发 现鹤壁地区的岩石圈为太古代地幔的浅部残留(Zheng 等, 2001, 2007; Tang等, 2013). 重力数据反演显示该区 域在60 160km深度内为负的密度扰动, 表现出较轻的 密度性质(Li等, 2011). 岩石学数据揭示, 在相同的温压 条件下克拉通的岩石圈地幔密度比非克拉通的岩石圈 地幔密度轻1.5 2.5\%(Poudjom Djomani等, 2001), 克拉 
通表现出低密度特征, $\sim 3.3 \mathrm{~g} \mathrm{~cm}^{-3}$ (Zhu R X等, 2012). 因此，推测高速、低密度的异常体H2”可能代表了难 熔的太古代岩石圈. 接收函数的结果表明该区域下方 存在复杂的分层结构，华北克拉通西部的古元古代板 片以 20倾角插入东部残留的太古代岩石圈内，在克 拉通破坏期间东部残留的太古代岩石圈内沿着如裂 隙、剪切带等薄弱带发生了氧化还原熔融, 形成小的 部分熔融带(Wang等，2013). 充斥于残留的太古代岩 石圈内的这些小的部分熔融带尺寸小于地震波分辨尺 度, 对地震波走时产生了一定影响, 这可能是造成该区 域高速异常幅值较低的原因.

\section{2 华北克拉通东部非均匀性破坏的动力学过程 启示}

本文的速度模型最显著的特征之一是华北克拉通 东部的上地幔表现出非均匀的小尺度横向速度结构特 征, 在60 250 km深度内速度模型不仅在横向上表现出 很好的构造相关性，在纵向上也表现出很好的连贯性 (图4a e 和5a e). 5.1.2节的分析表明，这些小尺度的速 度结构特征可能是华北克拉通东部上地幔遭受了非均 匀性破坏的结果. 因此, 新的科学问题是层析成像观测 到的华北克拉通东部非均匀性破坏现象的动力学指示 意义是什么?

层析成像观测到的华北克拉通下方地幔转换带内 的高速异常体(图4g h、5g h、6a g、k和 $b^{\prime} \sim g^{\prime} 、 k^{\prime}$ ) 被 认为是西向俯冲停滞的太平洋板片前沿(例如，Lebedev和Nolet，2003; Huang和Zhao，2006; Li等，2006, 2008; Tian等, 2009; Li和van der Hilst, 2010; Xu和Zhao, 2009; Feng等, 2010; Lei, 2012; Obrebski等, 2012; Zhao 等, 2012; Chen等, 2015). 本文的速度模型显示地幔转 换带内的高速异常体呈现多尺度横向变化的特征(图 $6 \mathrm{a} \sim \mathrm{g} 、 \mathrm{k}$ 和 $\left.\mathrm{b}^{\prime} \sim \mathrm{g}^{\prime} 、 \mathrm{k}^{\prime}\right)$, 由于纵、横波恢复能力的差异 (4.2节所述), 纵波和横波在地幔转换带内观测到的高 速异常体形态存在着一定的差异，但也显示了一些共 同的观测特征: 如果该高速异常体表示俯冲停滞的太 平洋板片, 则大洋板片的前沿位置位于 $118^{\circ} \mathrm{E}$ (图 $4 \mathrm{~g} \sim \mathrm{h}$ 和图 $5 \mathrm{~g} \sim \mathrm{h})$; 在 $116^{\circ} \sim 120^{\circ} \mathrm{E}$ 范围内，部分板片物质穿过 了 $660 \mathrm{~km}$ 间断面下沉到下地幔内(图 $6 \mathrm{~b} \sim \mathrm{f}$ 和 $\left.\mathrm{b}^{\prime} \sim \mathrm{f}^{\prime}\right)$. 高速 异常体非均一的形态特征提示俯冲的大洋板片内部可 能发生了尧曲变形，甚至局部断裂(Schmid等，2002; Ribe等, 2007; Čížková和Bina, 2013). 大洋板片俯冲到
富水的地幔转换带(Kuritani等，2011)内会引起脱水作 用, 脱水来自俯冲的板片, 或者地幔转换带, 或者来自 两者. 俯冲板片形态的改变(尧曲变形、断裂和局部下 沉等)也会加剧脱水作用. 动力学数值模拟实验(Yang 等，2017)表明，板片俯冲相关的脱水作用是空间非均 匀的, 脱水作用会引起地幔转换带顶部发生部分熔 融、触发湿上升流(较轻的含水/部分熔融熔体)上升, 而周围的地幔流场使垂向的湿上升流的方向偏转，这 种相互作用导致地幔流场的强烈非均匀性. 华北克拉 通东部上地幔内(200 410km深度)非均匀分布的低速 异常体 (图4e f、5e f和6)可能是较轻的部分熔融熔体 空间非均匀分布作用的结果.

华北克拉通中部下方的低纵、横波速度异常体 L1 异常幅值 $>2 \%$ 纵波速度扰动或 $>3 \%$ 横波速度扰动的 区域位于约60 250km深度, 呈NW-SE向，对于横波横 向上最大的尺度约 $400 \mathrm{~km} \times 100 \mathrm{~km}$ (图4a f、5a f、 $6 \mathrm{~b} 、 \mathrm{j}$ 和 $\left.\mathrm{a}^{\prime} \sim \mathrm{c}^{\prime} 、 \mathrm{~g}^{\prime} 、 \mathrm{~h}^{\prime} 、 \mathrm{j}^{\prime}\right) . V_{\mathrm{p}} / V_{\mathrm{s}}$ 的模型(Zhao等, 2012) 显示 $\mathrm{L} 1$ 区域的 $\delta \ln V_{\mathrm{s}} / \delta \ln V_{\mathrm{p}}$ 值为 2.4. 基于岩石物理实验 的正演计算(Cammarano等, 2003)表明, 上地幔内 $\delta \ln V_{\mathrm{s}} /$ $\delta \ln V_{\mathrm{p}}$ 随温度扰动的期望值从低温的 1.3 变化到高温的 2.2. 因此, L1区域的 $\delta \ln V_{\mathrm{s}} / \delta \ln V_{\mathrm{p}}$ 值可能反映了上地幔内 部分熔融作用的结果，异常幅值 $>2 \%$ 纵波速度扰动或 $>3 \%$ 横波速度扰动的区域低速异常对应于 1 2\%的部 分熔融(Hammond和Humphreys, 2000). 大同火山区域 碱性玄武岩(软流圈部分熔融体上涌的结果)和拉斑玄 武岩(部分熔融体与被侵蚀的、重富集的岩石圈地幔 作用后上涌的结果)的地理分布表现出与低速异常体 L1走向很好的一致性，从东南区域的拉斑玄武岩逐渐 过渡到西北区域的碱性玄武岩(Xu等，2005)。上升的 熔融体受岩石圈-软流圈界面起伏影响形成局部对流 (5.1.1节论述中，许多工作揭示了大同区域下方存在水 平向的地幔流动, 例如, Huang等, 2011; Zhao等, 2011; Chang等, 2012; Wang等, 2014)、引起小尺度上涌, 这 可能是该区域火山作用的起因(Xu等，2005; Wang和 Niu, 2011). 当对流的横向尺度较小时, 可能暗示大同 区域的火山作用起源于岩石圈-软流圈界面附近(Wang 和Niu，2011). 本文模型的纵切片图(图6b、j和 $\mathrm{a}^{\prime} \sim \mathrm{c}^{\prime}$ 、 $\mathrm{g}^{\prime} 、 \mathrm{~h}^{\prime} 、 \mathrm{j}^{\prime}$ )显示接收函数获取的岩石圈-软流圈界面形 态穿过 L 1 异常幅值较大的区域 $>2 \%$ 纵波速度扰动或 $>3 \%$ 横波速度扰动的区域)，这表明L1可能是脱水作用 造成的、来自地幔转换带的较轻部分熔融熔体随时间 
的推移在岩石圈-软流圈界面聚积的结果(Sakamaki等, 2013). 聚积的熔体与岩石圈地幔发生作用，导致岩石 圈地幔的重新富集. 华北克拉通中部下方的岩石圈地 幔表现出强烈的横向不均匀性，可能是多期次的熔融 或流体引起的太古宙地幔重新富集的结果(Tang等, 2013)。碱性玄武岩水含量和氧同位素组分的研究表 明，这些熔融或流体主要来自于俯冲的太平洋板片脱 水以及洋壳(夹杂海洋沉积)物质的贡献(Liu等，2015). 华北克拉通中部下方的动力学过程可能是华北克拉通 东部破坏过程的一个缩影.

动力学数值模拟实验表明，非均匀脱水引起的部 分熔融会引发上地幔内复杂的地幔流，因而克拉通内 部发育的岩浆事件不表现出明显的时空分布趋势 (Yang等, 2017). 从出露的岩浆事件分布(图7b)来看, 晚 二叠到晚侏罗的岩浆事件主要分布在华北克拉通四周 的造山带; 对应于克拉通破坏峰期的早白严岩浆事件 分布则从克拉通四周的造山带延伸到了克拉通的内 部，包括中央造山带以及郯庐断裂两侧(徐义刚等, 2009; Zhang等, 2014). 成像的模型显示环华北克拉通
四周的造山带、中央造山带和郯庐断裂的下方是显著 的低纵、横波速度异常(图 $4 \mathrm{a} \sim \mathrm{d}$ 和 $5 \mathrm{a} \sim \mathrm{d})$ ，这些区域的 低速异常分布与晚二叠至新生代出露的岩浆事件分布 有很好的空间对应关系(图7b), 这说明华北克拉通区 域的岩浆事件存在着明显的构造相关性. 由于动力学 数值模拟实验(Yang等，2017)未考虑岩石圈内部构造 (主要是碰撞造山缝合带和郯庐断裂)的影响, 岩浆事 件分布的构造相关性和动力学数值模拟实验结果的差 异表明，岩石圈内部构造在克拉通破坏过程中扮演了 重要作用(徐义刚等，2009; Chen，2010). 华北克拉通 区域金矿床的发育因与克拉通破坏的紧密联系被称为 “去克拉通化金矿床”，其分布与早白严岩浆事件有很 好的空间一致性，主要分布于沿着中央造山带和郯庐 断裂的两条NNE向的条带内(Zhu等，2015，2017), 岩 石圈内部的碰撞造山缝合带和郯庐断裂可能为成矿流 体的运移和富集提供了良好的空间.

综合以上分析，本文认为俯冲板片触发的湿上升 流导致的岩石圈重新富集是华北克拉通破坏的机制 (Wang等, 2015; Yang等, 2017). 图8是华北克拉通破坏

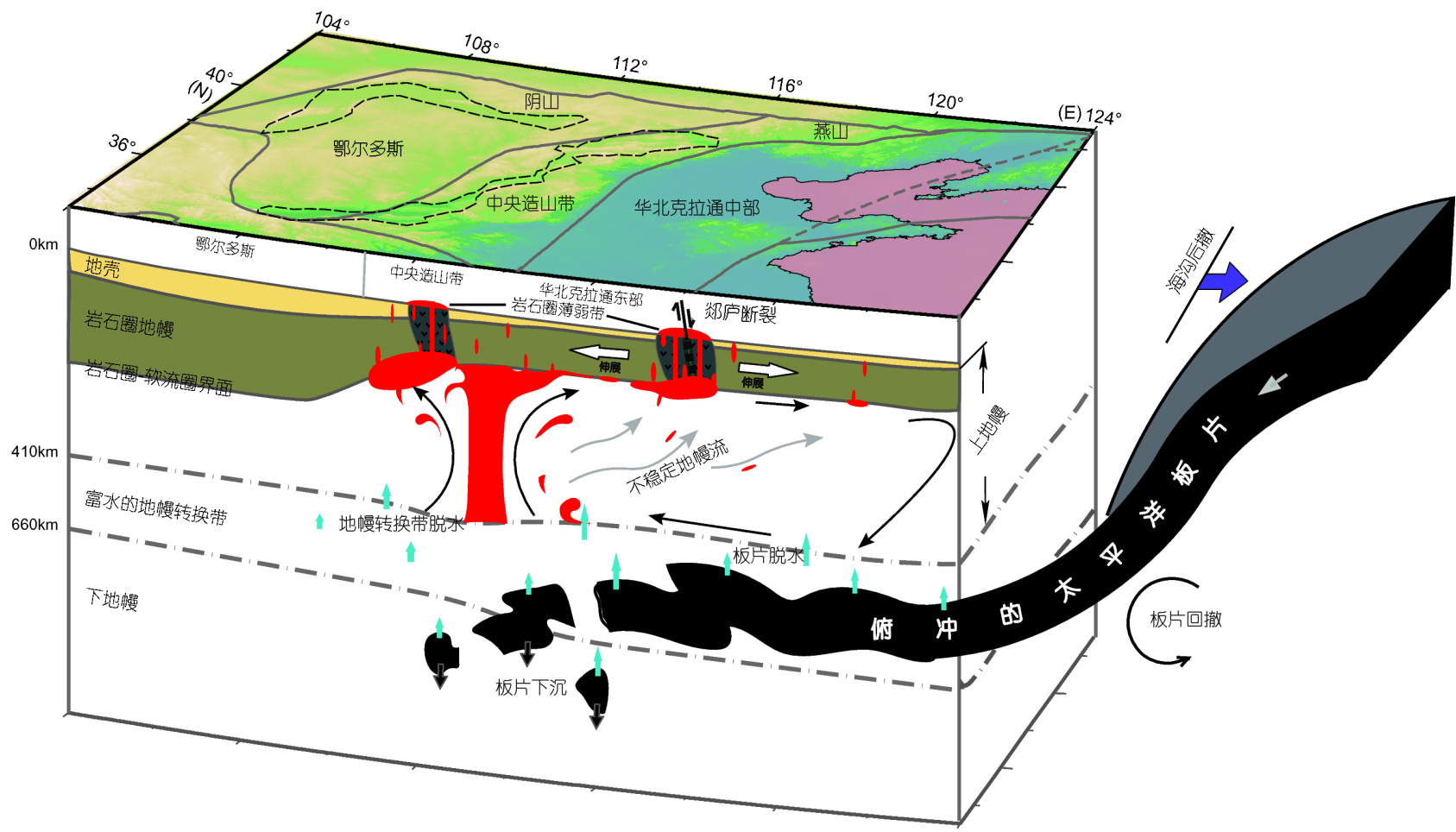

图 8 克拉通破坏示意图

该图参考Zhu等(2015)的模型修改，基于本文层析成像观测的结果，富水的地幔转换带(Kuritani等，2011)和板片脱水引起的湿地幔物质上涌 (Wang等, 2015; Yang等, 2017), 熔融体在岩石圈-软流圈界面聚积现象(Sakamaki等, 2013)以及金矿床(Zhu等, 2015, 2017)和中生代以来的岩 浆事件分布位置(徐义刚等, 2009; Zhang等, 2014) 
的动力学过程示意图. 在晚中生代, 俯冲到华北克拉通 下方的太平洋板片引起非均匀脱水作用，造成地幔转 换带顶部的地幔物质发生部分熔融、触发湿上升流. 湿上升流(较轻的含水/部分熔融熔体)的上升过程表现 出空间的非均匀性, 并引起上地幔内(200 410km深度) 复杂的地幔流动. 较轻的熔体遇到稳定的克拉通岩石 圈根, 在岩石圈-软流圈界面发生聚积. 在浮力作用下, 部分熔体可能通过岩石圈内相对薄弱区域(碰撞造山 缝合带或断裂区域)继续上升. 因此，华北克拉通东部 表现出的非均匀性破坏特征可能是上地幔内非均匀分 布的部分熔融熔体和克拉通岩石圈内固有的部构造薄 弱带两个因素共同作用、演化的结果.

\section{3 苏鲁超高压变质带上地幔速度结构特征}

本文的速度模型观测到苏鲁造山带下方的高纵、 横波速度异常体 $\mathrm{H} 3$ 在 $200 \mathrm{~km}$ 深度内构造上似乎受控于 烟台-青岛-五莲断裂和郯庐断裂(图 $4 \mathrm{a} \sim \mathrm{d}$ 和 $5 \mathrm{a} \sim \mathrm{d}$ ). 苏鲁 造山带和大别造山带之间沿㸚庐断裂 $550 \mathrm{~km}$ 的左旋 滑距是由炏庐断裂自中生代同碰撞转换断层发育为新 生代走滑断层的长期演化结果(Zhu等, 2005, 2009). 高 速异常体H3形态上呈北-东-北向, 西侧的异常幅值较 高, 向下延伸得更深 $300 \mathrm{~km}$ (图4a f), 可能和扬子克拉 通在三叠纪与华北克拉通碰撞过程中的逆时针旋转有 关(Gilder等, 1999; Zhu G等, 2009). 高压-超高压变质 岩的出露位置和岩石学的证据表明苏鲁造山带属于扬 子克拉通的一部分(Faure等, 2003). 对莒南晚白严玄武 岩的研究, 发现存在高镁地幔橄榄岩捕掳体, 暗示下方 可能存在古老岩石圈地幔的残留(Ying等, 2006). 高速 异常体H3可能代表了往北西向俯冲的扬子克拉通的 残留, 烟台-青岛-五莲断裂可能是扬子克拉通俯冲的 北缘位置.

\section{6 结论}

通过有限频多频段联合反演方法，本文获得了华 北克拉通区域精细的三维纵、横波速度模型. 新的速 度模型呈现明显的横向不均匀性，与地质构造单元存 在很好的空间对应关系:

(1) 鄂尔多斯块体下方是延伸到 $300 \mathrm{~km}$ 深度左右 的高纵、横波速度异常，代表了古老的克拉通岩石圈 根. 而环鄂尔多斯新生代的银川-河套裂谷和山西-陕
西裂谷下方则呈现低纵、横波速度异常，可能是该区 域新生代构造活化的结果.

（2）华北克拉通中部整体呈现为低纵、横波速度 异常, 异常幅值显著强于东部, 且存在南北空间差异. 北部的低速异常体对应于浅表的第四纪火山群(例如, 大同火山), 呈NW-SE走向, 向下延伸到地幔转换带深 度. 南部的低速异常体则只延伸到约200 300km深度. 多学科证据表明华北克拉通中部的低速异常与太平洋 板片的俯冲密切相关. 临近山西-陕西裂谷的转换拉伸 区域，在南、北低速异常体之间可以观测到一小块显 著的高速异常体.

(3) 华北克拉通东部的上地幔呈现较中、西部非 均匀的小尺度横向速度结构特征，暗示了华北克拉通 东部破坏的空间非均匀性. 㸚庐断裂的下方是延伸到 约200 300km深度的低纵、横波速度异常. 其西侧的 南缘和北缘燕山地区下方是高纵、横波速度异常, 异 常幅值不高, 可能代表了残留的克拉通岩石圈. 其东 侧苏鲁造山带下方观测到的高纵、横波速度异常体延 伸到约 $300 \mathrm{~km}$ 深度, 高速异常体的北缘与烟台-青岛-五 莲断裂吻合, 西缘与郯庐断裂吻合.

(4) 地幔转换带内的高速异常体表现出非均一的 形态特征，代表了俯冲停滞的太平洋板片前沿，前沿 位于 $118^{\circ} \mathrm{E}$. 部分高速异常体穿过 $660 \mathrm{~km}$ 间断面, 主要 位于 $116^{\circ} \sim 120^{\circ} \mathrm{E}$ 区间.

新的成像模型主要综合接收函数的观测(Chen, 2010; 郑天愉等，2017)、浅部地质的观测(徐义刚等, 2009; Zhang等，2014)、金矿床的研究(Zhu等，2015, 2017)以及动力学数值模拟实验(Yang等, 2017)进行分 析，本文认为华北克拉通东部呈现的小尺度横向速度 结构特征是太平洋板片俯冲诱导的湿上升流和克拉通 岩石圈内部固有的构造薄弱带(例如, 碰撞造山缝合带 和郯庐断裂)共同作用的结果. 本文模型中苏鲁超高压 变质带位于研究区域的边缘, 分辨率测试显示了该区 域速度结构的可靠性. 但该区域更精细的速度模型对 深入认识郯庐断裂的发育、华北和华南碰撞过程有重 要动力学意义, 有待进一步的工作.

致谢感谢中国地震局地球物理研究所的郑秀芬研究 员、中国科学院地质与地球物理研究所地震实验室同事 及北京大学同仁对地震数据的收集、整理. 感谢袁怀玉 博士、梁晓峰博士和张风雪博士的有益建议, 感谢张耀 
阳博士提供最新的岩石圈-软流圈界面深度数据. 感谢主 编、编委和三位若名审稿人建设性的评审意见. 波形数 据由中国科学院地质与地球物理研究所地震实验室和中 国地震局地球物理研究所中国国家地震台网数据管理中 心提供.

\section{参考文献}

邓晋福, 莫宣学, 赵海玲, 罗照华, 杜杨松. 1994. 中国东部岩石圈根/ 去根作用与大陆“活化”一东亚型大陆动力学模式研究计划. 现 代地质, (3): 349-356

何丽娟, 胡圣标, 汪集旸. 2001. 中国东部大陆地区岩石圈热结构特 征. 自然科学进展, 11: 966-969

李洪颜, 徐义刚, 黄小龙, 何斌, 罗震宇, 燕滨. 2009. 华北克拉通北缘 晚古生代活化: 山西宁武-静乐盆地上石炭统太原组碎屑锆石U$\mathrm{Pb}$ 测年及Hf 同位素证据. 科学通报, 54: 632-640

吴福元, 徐义刚, 高山, 郑建平. 2008. 华北岩石圈减薄与克拉通破坏 研究的主要学术争论. 岩石学报, 24: 1145-1174

吴福元, 徐义刚, 朱日祥, 张国伟. 2014. 克拉通岩石圈减薄与破坏. 中国科学: 地球科学, 44: 2358-2372

徐义刚, 李洪颜, 庞崇进, 何斌. 2009. 论华北克拉通破坏的时限. 科 学通报, 54: 1974-1989

王乃梁, 杨景春, 夏正楷, 莫多闻, 李有利, 潘森. 1996. 山西地堑系新 生代沉积与构造地貌. 北京: 科学出版社

王敏, 沈正康, 牛之俊, 张祖胜, 孙汉荣, 甘卫军, 王琪, 任群. 2003. 现 今中国大陆地壳运动与活动块体模型. 中国科学 $\mathrm{D}$ 辑: 地球科学, S1: $21-32$

汪集旸, 黄少鹏, 陈墨香. 1996. 大地热流圈. 见: 袁学成. 中国地球物 理图集. 北京: 地质出版社. 102

郑天愉, 段永红, 许卫卫, 艾印双, 陈凌, 赵亮, 张耀阳, 徐小兵. 2017. 华北地区地壳-上地幔地震波速度结构模型v2.0, http://www.craton.cn/data

赵越, 陈斌, 张拴宏, 刘建民, 胡健民, 刘健, 裴军令. 2010. 华北克拉 通北缘及邻区前燕山期主要地质事件. 中国地质, 37: 900-915

朱日祥, 徐义刚, 朱光, 张宏福, 夏群科, 郑天愉. 2012. 华北克拉通破 坏. 中国科学: 地球科学, 42: 1135-1159

Anderson D L, Sammis C. 1970. Partial melting in the upper mantle. Phys Earth Planet Inter, 3: 41-50

Allen R M, Nolet G, Morgan W J, Vogfjörd K, Bergsson B H, Erlendsson P, Foulger G R, Jakobsdóttir S, Julian B R, Pritchard M, Ragnarsson S, Stefánsson R. 2002. Imaging the mantle beneath Iceland using integrated seismological techniques. J Geophys Res, 107: $3-1-3-16$

Artemieva I M, Billien M, Lévêque J J, Mooney W D. 2004. Shear wave velocity, seismic attenuation, and thermal structure of the continental upper mantle. Geophys J Int, 157: 607-628

Artemieva I M, Mooney W D. 2001. Thermal thickness and evolution of Precambrian lithosphere: A global study. J Geophys Res, 106: $16387-16414$

Bao X, Xu M, Wang L, Mi N, Yu D, Li H. 2011. Lithospheric structure of the Ordos Block and its boundary areas inferred from Rayleigh wave dispersion. Tectonophysics, 499: 132-141

Cammarano F, Goes S, Vacher P, Giardini D. 2003. Inferring uppermantle temperatures from seismic velocities. Phys Earth Planet Inter, 138: 197-222

Chang L, Wang C Y, Ding Z. 2012. Upper mantle anisotropy beneath North China from shear wave splitting measurements. Tectonophysics, 522-523: 235-242

Chen L. 2010. Concordant structural variations from the surface to the base of the upper mantle in the North China Craton and its tectonic implications. Lithos, 120: 96-115

Chen L, Ai Y S. 2009. Discontinuity structure of the mantle transition zone beneath the North China Craton from receiver function migration. J Geophys Res, 114: B06307

Chen L, Jiang M, Yang J, Wei Z, Liu C, Ling Y. 2014. Presence of an intralithospheric discontinuity in the central and western North China Craton: Implications for destruction of the craton. Geology, 42: $223-226$

Chen L, Tao W, Zhao L, Zheng T Y. 2008. Distinct lateral variation of lithospheric thickness in the Northeastern North China Craton. Earth Planet Sci Lett, 267: 56-68

Chen L, Zheng T, Xu W. 2006. A thinned lithospheric image of the Tanlu Fault Zone, eastern China: Constructed from wave equation based receiver function migration. J Geophys Res, 111: B09312

Chen M, Niu F, Liu Q, Tromp J, Zheng X. 2015. Multiparameter adjoint tomography of the crust and upper mantle beneath East Asia: 1. Model construction and comparisons. J Geophys Res-Solid Earth, 120: $1762-1786$

Chevrot S, Villaseñor A, Sylvander M, Benahmed S, Beucler E, Cougoulat G, Delmas P, de Saint Blanquat M, Diaz J, Gallart J, Grimaud F, Lagabrielle Y, Manatschal G, Mocquet A, Pauchet H, Paul A, Péquegnat C, Quillard O, Roussel S, Ruiz M, Wolyniec D. 2014. High-resolution imaging of the Pyrenees and Massif Central from the data of the PYROPE and IBERARRAY portable array deployments. J Geophys Res-Solid Earth, 119: 6399-6420

Č́źžková H, Bina C R. 2013. Effects of mantle and subduction-interface rheologies on slab stagnation and trench rollback. Earth Planet Sci Lett, 379: 95-103

Dahlen F A, Hung S H, Nolet G. 2000. Fréchet kernels for finitefrequency traveltimes-I. Theory. Geophys J Int, 141: 157-174

Davis G A. 2003. The Yanshan belt of North China: Tectonics, adakitic 
magmatism, and crustal evolution. Earth Sci Front, 10: 373-384

Davies J H. 2013. Global map of solid Earth surface heat flow. Geochem Geophys Geosyst, 14: 4608-4622

Davis G A, Zheng Y, Wang C, Darby B J, Zhang C, Gehrels G. 2001. Mesozoic tectonic evolution of the Yanshan fold and thrust belt, with emphasis on Hebei and Liaoning provinces, northern China. In: Hendrix M S, Davis G A, eds. Paleozoic and Mesozoic Tectonic Evolution of Central and Eastern Asia. Mem Geol Soc Am, 194: 171-197

Ekström G, Dziewonski A M. 1998. The unique anisotropy of the Pacific upper mantle. Nature, 394: 168-172

Faure M, Lin W, Monié P, Le Breton N, Poussineau S, Panis D, Deloule E. 2003. Exhumation tectonics of the ultrahigh-pressure metamorphic rocks in the Qinling orogen in east China: New petrological-structural-radiometric insights from the Shandong Peninsula. Tectonics, 22: 1018

Feng M, van der Lee S, An M J, Zhao Y. 2010. Lithospheric thickness, thinning, subduction, and interaction with the asthenosphere beneath China from the joint inversion of seismic S-wave train fits and Rayleigh-wave dispersion curves. Lithos, 120: 116-130

Gao S, Rudnick R L, Yuan H L, Liu X M, Liu Y S, Xu W L, Ling W L, Ayers J, Wang X C, Wang Q H. 2004. Recycling lower continental crust in the North China craton. Nature, 432: 892-897

Gilder S A, Leloup P H, Courtillot V, Chen Y, Coe R S, Zhao X, Xiao W, Halim N, Cogné J P, Zhu R. 1999. Tectonic evolution of the Tancheng-Lujiang (Tan-Lu) fault via Middle Triassic to Early Cenozoic paleomagnetic data. J Geophys Res, 104: 15365-15390

Goes S, Govers R, Vacher P. 2000. Shallow mantle temperatures under Europe from $\mathrm{P}$ and $\mathrm{S}$ wave tomography. J Geophys Res, 105: 11153-11169

Griffin W L, Andi Z, O'Reilly S Y, Ryan C G. 1998. Phanerozoic evolution of the lithosphere beneath the Sino-Korean craton. Mantle Dynamics and Plate Interactions in East Asia. 107-126

Gripp A E, Gordon R G. 2002. Young tracks of hotspots and current plate velocities. Geophys J Int, 150: 321-361

Guillot S, Hattori K, Agard P, Schwartz S, Vidal O. 2009. Exhumation processes in oceanic and continental subduction contexts: A review. In: Lallemand S, Funiciello F, eds. Subduction Zone Geodynamics. Berlin: Springer. 175-205

Hammond W C, Humphreys E D. 2000. Upper mantle seismic wave velocity: Effects of realistic partial melt geometries. J Geophys Res, 105: 10975-10986

Hieronymus C F, Shomali Z H, Pedersen L B. 2007. A dynamical model for generating sharp seismic velocity contrasts underneath continents: Application to the Sorgenfrei-Tornquist Zone. Earth Planet Sci Lett, 262: 77-91
Hu S B, He L J, Wang J Y. 2000. Heat flow in the continental area of China: A new data set. Earth Planet Sci Lett, 179: 407-419

Hu S B, He L J, Wang J Y. 2001. Compilation of heat flow data in the China continental area. Chin J Geophys, 44: 604-618

Huang J L, Zhao D P. 2004. Crustal heterogeneity and seismotectonics of the region around Beijing, China. Tectonophysics, 385: 159-180

Huang J L, Zhao D P. 2006. High-resolution mantle tomography of China and surrounding regions. J Geophys Res, 111: B09305

Huang Z, Wang L, Zhao D, Mi N, Xu M. 2011. Seismic anisotropy and mantle dynamics beneath China. Earth Planet Sci Lett, 306: 105117

Hung S H, Dahlen F A, Nolet G. 2000. Fréchet kernels for finitefrequency traveltimes-II. Examples. Geophys J Int, 141: 175-203

Hung S H, Shen Y, Chiao L Y. 2004. Imaging seismic velocity structure beneath the Iceland hot spot: A finite frequency approach. J Geophys Res, 109: B08305

Jiang M M, Ai Y S, Chen L, Yang Y J. 2013. Local modification of the lithosphere beneath the central and western North China Craton: 3D constraints from Rayleigh wave tomography. Gondwana Res, 24 : 849-864

Karato S I, Karki B B. 2001. Origin of lateral variation of seismic wave velocities and density in the deep mantle. J Geophys Res, 106: 21771-21783

Kennett B L N, Engdahl E R. 1991. Traveltimes for global earthquake location and phase identification. Geophys J Int, 105: 429-465

Koulakov I, Kaban M K, Tesauro M, Cloetingh S. 2009. P- and $S$ velocity anomalies in the upper mantle beneath Europe from tomographic inversion of ISC data. Geophys J Int, 179: 345-366

Kuritani T, Ohtani E, Kimura J I. 2011. Intensive hydration of the mantle transition zone beneath China caused by ancient slab stagnation. Nat Geosci, 4: 713-716

Lebedev S, Nolet G. 2003. Upper mantle beneath Southeast Asia from $S$ velocity tomography. J Geophys Res, 108: 2048

Lei J. 2012. Upper-mantle tomography and dynamics beneath the North China Craton. J Geophys Res, 117: B06313

Lei J, Xie F, Lan C, Xing C, Ma S. 2008. Seismic images under the Beijing region inferred from $\mathrm{P}$ and PmP data. Phys Earth Planet Inter, 168: 134-146

Lei J, Xie F, Fan Q, Santosh M. 2013. Seismic imaging of the deep structure under the Chinese volcanoes: An overview. Phys Earth Planet Inter, 224: 104-123

Lenardic A, Moresi L, Mühlhaus H. 2000. The role of mobile belts for the longevity of deep cratonic lithosphere: The crumple zone model. Geophys Res Lett, 27: 1235-1238

Lenardic A, Moresi L N, Mühlhaus H. 2003. Longevity and stability of cratonic lithosphere: Insights from numerical simulations of coupled 
mantle convection and continental tectonics. J Geophys Res, 108: 2303

Li C, van der Hilst R D. 2010. Structure of the upper mantle and transition zone beneath Southeast Asia from traveltime tomography. J Geophys Res, 115: B07308

Li C, van der Hilst R D, Engdahl E R, Burdick S. 2008. A new global model for $\mathrm{P}$ wave speed variations in Earth's mantle. Geochem Geophys Geosyst, 9: Q05018

Li C, van der Hilst R D, Toksöz M N. 2006. Constraining P-wave velocity variations in the upper mantle beneath Southeast Asia. Phys Earth Planet Inter, 154: 180-195

Li S G, Xiao Y L, Liou D L, Chen Y Z, Ge N J, Zhang Z Q, Sun S S, Cong B L, Zhang R Y, Hart S R, Wang S S. 1993. Collision of the North China and Yangtse Blocks and formation of coesite-bearing eclogites: Timing and processes. Chem Geol, 109: 89-111

Li Y, Yang Y. 2011. Gravity data inversion for the lithospheric density structure beneath North China Craton from EGM 2008 model. Phys Earth Planet Inter, 189: 9-26

Li Y, Wu Q, Pan J, Zhang F, Yu D. 2013. An upper-mantle S-wave velocity model for East Asia from Rayleigh wave tomography. Earth Planet Sci Lett, 377-378: 367-377

Liang X, Shen Y, Chen Y J, Ren Y. 2011. Crustal and mantle velocity models of southern Tibet from finite frequency tomography. J Geophys Res, 116: B02408

Liu D Y, Nutman A P, Compston W, Wu J S, Shen Q H. 1992 Remnants of $\geq 3800$ Ma crust in the Chinese part of the Sino-Korean craton. Geology, 20: 339-342

Liu J, Xia Q K, Deloule E, Ingrin J, Chen H, Feng M. 2015. Water content and oxygen isotopic composition of alkali basalts from the Taihang Mountains, China: Recycled oceanic components in the mantle source. J Petrol, 56: 681-702

Meyerholtz K A, Pavlis G L, Szpakowski S A. 1989. Convolutional quelling in seismic tomography. Geophysics, 54: 570-580

Nolet G. 1985. Solving or resolving inadequate and noisy tomographic systems. J Comput Phys, 61: 463-482

Obrebski M, Allen R M, Zhang F X, Pan J T, Wu Q J, Hung S H. 2012. Shear wave tomography of China using joint inversion of body and surface wave constraints. J Geophys Res, 117: B01311

Paige C C, Saunders M A. 1982. LSQR: An algorithm for sparse linear equations and sparse least squares. ACM Trans Math Softw, 8: 4371

Pan G T, Mo X X, Hou Z Q, Zhu D C, Wang L Q, Li G M, Zhao Z D, Geng Q R, Liao Z L. 2006. Spatial-temporal framework of the Gangdese Orogenic Belt and its evolution. Acta Petrol Sin, 22: 521533

Polet J, Anderson D L. 1995. Depth extent of cratons as inferred from tomographic studies. Geology, 23: 205-208

Poudjom Djomani Y H, O’Reilly S Y, Griffin W L, Morgan P. 2001. The density structure of subcontinental lithosphere through time. Earth Planet Sci Lett, 184: 605-621

Ren Y, Shen Y. 2008. Finite frequency tomography in southeastern Tibet: Evidence for the causal relationship between mantle lithosphere delamination and the north-south trending rifts. J Geophys Res, 113: B10316

Ribe N M, Stutzmann E, Ren Y, van der Hilst R. 2007. Buckling instabilities of subducted lithosphere beneath the transition zone. Earth Planet Sci Lett, 254: 173-179

Rocha M P, Schimmel M, Assumpção M. 2011. Upper-mantle seismic structure beneath SE and Central Brazil from P- and S-wave regional traveltime tomography. Geophys J Int, 184: 268-286

Rychert C A, Fischer K M, Rondenay S. 2005. A sharp lithosphereasthenosphere boundary imaged beneath eastern North America. Nature, 436: 542-545

Sakamaki T, Suzuki A, Ohtani E, Terasaki H, Urakawa S, Katayama Y, Funakoshi K I, Wang Y, Hernlund J W, Ballmer M D. 2013. Ponded melt at the boundary between the lithosphere and asthenosphere. Nat Geosci, 6: 1041-1044

Schmandt B, Humphreys E. 2010. Complex subduction and small-scale convection revealed by body-wave tomography of the western United States upper mantle. Earth Planet Sci Lett, 297: 435-445

Schmid C, Goes S, van der Lee S, Giardini D. 2002. Fate of the Cenozoic Farallon slab from a comparison of kinematic thermal modeling with tomographic images. Earth Planet Sci Lett, 204: 1732

Shen Z K, Zhao C, Yin A, Li Y, Jackson D D, Fang P, Dong D. 2000. Contemporary crustal deformation in east Asia constrained by Global Positioning System measurements. J Geophys Res, 105: $5721-5734$

Sobolev S V, Zeyen H, Stoll G, Werling F, Altherr R, Fuchs K. 1995. Upper mantle temperatures from teleseismic tomography of French Massif Central including effects of composition, mineral reactions, anharmonicity, anelasticity and partial melt. Earth Planet Sci Lett, 139: $147-163$

Sun Y S, Toksöz M N. 2006. Crustal structure of China and surrounding regions from $\mathrm{P}$ wave traveltime tomography. J Geophys Res, 111: B03310

Sun Y S, Toksöz M N, Pei S P, Zhao D, Morgan F D, Rosca A. 2008a. $\mathrm{S}$ wave tomography of the crust and uppermost mantle in China. J Geophys Res, 113: B11307

Sun Y S, Toksoz M N, Pei S P, Morgan F D. 2008b. The layered shearwave velocity structure of the crust and uppermost mantle in China. Bull Seismol Soc Am, 98: 746-755 
Tang Q, Chen L. 2008. Structure of the crust and uppermost mantle of the Yanshan Belt and adjacent regions at the northeastern boundary of the North China Craton from Rayleigh Wave Dispersion Analysis. Tectonophysics, 455: 43-52

Tang Y J, Zhang H F, Ying J F. 2006. Asthenosphere-lithospheric mantle interaction in an extensional regime: Implication from the geochemistry of Cenozoic basalts from Taihang Mountains, North China Craton. Chem Geol, 233: 309-327

Tang Y J, Zhang H F, Ying J F, Su B X, Chu Z Y, Xiao Y, Zhao X M. 2013. Highly heterogeneous lithospheric mantle beneath the Central Zone of the North China Craton evolved from Archean mantle through diverse melt refertilization. Gondwana Res, 23: 130-140

Tian Y, Zhao D. 2011. Destruction mechanism of the North China Craton: Insight from $\mathrm{P}$ and $\mathrm{S}$ wave mantle tomography. J Asian Earth Sci, 42: 1132-1145

Tian Y, Zhao D P, Sun R M, Teng J W. 2009. Seismic imaging of the crust and upper mantle beneath the North China Craton. Phys Earth Planet Inter, 172: 169-182

Van Decar J C, Crosson R S. 1990. Determination of teleseismic relative phase arrival times using multi-channel cross-correlation and least squares. Bull Seismol Soc of Amer, 80: 150-169

Wang J, Wu H, Zhao D P. 2014. P wave radial anisotropy tomography of the upper mantle beneath the North China Craton. Geochem Geophys Geosyst, 15: 2195-2210

Wang P, Xu M, Wang L, Mi N, Yu D, Li H, Li C, Bao X, Huang T, Huang H. 2013. Seismic evidence for the stratified lithosphere in the south of the North China Craton. J Geophys Res-Solid Earth, 118: $570-582$

Wang Q, Zhang P Z, Freymueller J T, Bilham R, Larson K M, Lai X, You X, Niu Z, Wu J, Li Y, Liu J, Yang Z, Chen Q. 2001. Present-day crustal deformation in China constrained by global positioning system measurements. Science, 294: 574-577

Wang X C, Wilde S A, Li Q L, Yang Y N. 2015. Continental flood basalts derived from the hydrous mantle transition zone. Nat Commun, 6: 7700

Wang X L, Niu F L. 2011. Imaging the mantle transition zone beneath eastern and central China with CEArray receiver functions. Earthq Sci, 24: $65-75$

Wang Y. 2001. Heat flow pattern and lateral variations of lithosphere strength in China mainland: Constraints on active deformation. Phys Earth Planet Inter, 126: 121-146

Wawerzinek B, Ritter J R R, Roy C. 2013. New constraints on the 3D shear wave velocity structure of the upper mantle underneath Southern Scandinavia revealed from non-linear tomography. Tectonophysics, 602: 38-54

Wei W, Xu J, Zhao D, Shi Y. 2012. East Asia mantle tomography: New insight into plate subduction and intraplate volcanism. J Asian Earth Sci, 60: $88-103$

Xiao W J, Windley B F, Hao J, Zhai M G. 2003. Accretion leading to collision and the Permian Solonker suture, Inner Mongolia, China: Termination of the central Asian orogenic belt. Tectonics, 22: 1069 Xiao W J, Windley B F, Yong Y, Yan Z, Yuan C, Liu C, Li J. 2009. Early Paleozoic to Devonian multiple-accretionary model for the Qilian Shan, NW China. J Asian Earth Sci, 35: 323-333

Xu P, Liu F, Ye K, Wang Q, Cong B, Chen H. 2002. Flake tectonics in the Sulu orogen in eastern China as revealed by seismic tomography. Geophys Res Lett, 29: 23-1-23-4

Xu P F, Zhao D P. 2009. Upper-mantle velocity structure beneath the North China Craton: Implications for lithospheric thinning. Geophys J Int, 177: 1279-1283

Xu W W, Zheng T Y, Zhao L. 2011. Mantle dynamics of the reactivating North China Craton: Constraints from the topographies of the 410-km and 660-km discontinuities. Sci China Earth Sci, 54: $881-887$

Xu Y G. 2001. Thermo-tectonic destruction of the archaean lithospheric keel beneath the sino-korean craton in china: Evidence, timing and mechanism. Phys Chem Earth Part A-Solid Earth Geodesy, 26: 747757

Xu Y G. 2007. Diachronous lithospheric thinning of the North China Craton and formation of the Daxin'anling-Taihangshan gravity lineament. Lithos, 96: 281-298

Xu Y G, Blusztajn J, Ma J L, Suzuki K, Liu J F, Hart S R. 2008. Late Archean to Early Proterozoic lithospheric mantle beneath the western North China craton: Sr-Nd-Os isotopes of peridotite xenoliths from Yangyuan and Fansi. Lithos, 102: 25-42

Xu Y G, Chung S L, Ma J, Shi L. 2004. Contrasting Cenozoic lithospheric evolution and architecture in the western and eastern Sino-Korean Craton: Constraints from geochemistry of basalts and mantle xenoliths. J Geol, 112: 593-605

Xu Y G, Ma J L, Frey F A, Feigenson M D, Liu J F. 2005. Role of lithosphere-asthenosphere interaction in the genesis of Quaternary alkali and tholeiitic basalts from Datong, western North China Craton. Chem Geol, 224: 247-271

Yang J, Zhao L, Kaus B J P, Lu G, Wang K, Zhu R. 2017. Slabtriggered wet upwellings produce large volumes of melt: Insights into the destruction of the North China Craton. Tectonophysics, doi: 10.1016/j.tecto.2017.04.009

Yin A, Nie S Y. 1996. A Phanerozoic palinspastic reconstruction of China and its neighboring regions. In: Yin A, Harrison M, eds. The Tectonic Evolution of Asia. Cambridge: Cambridge University Press. $442-484$

Ying J, Zhang H, Kita N, Morishita Y, Shimoda G. 2006. Nature and 
evolution of Late Cretaceous lithospheric mantle beneath the eastern North China Craton: Constraints from petrology and geochemistry of peridotitic xenoliths from Jünan, Shandong Province, China. Earth Planet Sci Lett, 244: 622-638

Zhang H F. 2009. Peridotite-melt interaction: A key point for the destruction of cratonic lithospheric mantle. Chin Sci Bull, 54: 3417 3437

Zhang K J. 1997. North and South China collision along the eastern and southern North China margins. Tectonophysics, 270: 145-156

Zhang M, Zhou X H, Zhang J B. 1998. Nature of the lithospheric mantle beneath NE China: Evidence from potassic volcanic rocks and mantle xenoliths. In: Griffin W L, Zhang A D, O'Reilly S Y, eds. Mantle Dynamics and Plate Interactions in East Asia. 197-219

Zhang S H, Zhao Y, Davis G A, Ye H, Wu F. 2014. Temporal and spatial variations of Mesozoic magmatism and deformation in the North China Craton: Implications for lithospheric thinning and decratonization. Earth-Sci Rev, 131: 49-87

Zhang Y, Wang Y, Zhao L, Jin Z. 2016. On velocity anomalies beneath southeastern China: An investigation combining mineral physics studies and seismic tomography observations. Gondwana Res, 31: 200-217

Zhang Y Q, Mercier J L, Vergély P. 1998. Extension in the graben systems around the Ordos (China), and its contribution to the extrusion tectonics of south China with respect to Gobi-Mongolia. Tectonophysics, 285: 41-75

Zhang Y Q, Ma Y S, Yang N, Shi W, Dong S. 2003. Cenozoic extensional stress evolution in North China. J Geodyn, 36: 591-613

Zhao D. 2007. Seismic images under 60 hotspots: Search for mantle plumes. Gondwana Res, 12: 335-355

Zhao D, Hasegawa A, Kanamori H. 1994. Deep structure of Japan subduction zone as derived from local, regional, and teleseismic events. J Geophys Res, 99: 22313-22329

Zhao D P, Isozaki Y, Maruyama S. 2017. Seismic imaging of the Asian orogens and subduction zones. J Asian Earth Sci, 145: 349-367

Zhao D P, Lei J S, Tang R. 2004. Origin of the Changbai intraplate volcanism in Northeast China: Evidence from seismic tomography. Chin Sci Bull, 49: 1401-1408

Zhao G, Wilde S A, Cawood P A, Sun M. 2001. Archean blocks and their boundaries in the North China Craton: Lithological, geochemical, structural and $P-T$ path constraints and tectonic evolution. Precambrian Res, 107: 45-73

Zhao G C, Sun M, Wilde S A. 2003. Major tectonic units of the North China Craton and their Paleoproterozoic assembly. Sci China Ser DEarth Sci, 46: 23-38

Zhao L, Allen R M, Zheng T, Hung S H. 2009. Reactivation of an Archean craton: Constraints from P- and S-wave tomography in
North China. Geophys Res Lett, 36: L17306

Zhao L, Allen R M, Zheng T, Zhu R. 2012. High-resolution body wave tomography models of the upper mantle beneath eastern China and the adjacent areas. Geochem Geophys Geosyst, 13: Q06007

Zhao L, Paul A, Malusà M G, Xu X, Zheng T, Solarino S, Guillot S, Schwartz S, Dumont T, Salimbeni S, Aubert C, Pondrelli S, Wang Q, Zhu R. 2016. Continuity of the Alpine slab unraveled by highresolution P wave tomography. J Geophys Res-Solid Earth, 121: $8720-8737$

Zhao L, Xu X, Malusà M G. 2017. Seismic probing of continental subduction zones. J Asian Earth Sci, 145: 37-45

Zhao L, Xue M. 2010. Mantle flow pattern and geodynamic cause of the North China Craton reactivation: Evidence from seismic anisotropy. Geochem Geophys Geosyst, 11: Q07010

Zhao L, Zheng T, Lu G, Ai Y. 2011. No direct correlation of mantle flow beneath the North China Craton to the India-Eurasia collision: Constraints from new SKS wave splitting measurements. Geophys J Int, 187: 1027-1037

Zhao X M, Zhang H F, Su F, Hu Z C, Lo C H, Wang Y, Yang S H, Guo J H. 2013. Phlogopite ${ }^{40} \mathrm{Ar} /{ }^{39} \mathrm{Ar}$ geochronology of mantle xenoliths from the North China Craton: Constraints on the eruption ages of Cenozoic basalts. Gondwana Res, 23: 208-219

Zheng J, O'Reilly S Y, Griffin W L, Lu F, Zhang M, Pearson N J. 2001. Relict refractory mantle beneath the eastern North China block: Significance for lithosphere evolution. Lithos, 57: 43-66

Zheng J P, Griffin W L, O’Reilly S Y, Yu C M, Zhang H F, Pearson N, Zhang M. 2007. Mechanism and timing of lithospheric modification and replacement beneath the eastern North China Craton: Peridotitic xenoliths from the 100 Ma Fuxin basalts and a regional synthesis. Geochim Cosmochim Acta, 71: 5203-5225

Zheng T Y, Zhao L, Xu W W, Zhu R X. 2008. Insight into modification of North China Craton from seismological study in the Shandong Province. Geophys Res Lett, 35: L22305

Zheng X F, Yao Z X, Liang J H, Zheng J. 2010. The role played and opportunities provided by IGP DMC of China National seismic network in Wenchuan Earthquake disaster relief and researches. Bull Seismol Soc Am, 100: 2866-2872

Zhu G, Wang Y, Liu G, Niu M, Xie C, Li C. $2005 .{ }^{40} \mathrm{Ar} /{ }^{39} \mathrm{Ar}$ dating of strike-slip motion on the Tan-Lu fault zone, East China. J Struct Geol, 27: 1379-1398

Zhu G, Liu G S, Niu M L, Xie C L, Wang Y S, Xiang B. 2009. Syncollisional transform faulting of the Tan-Lu fault zone, East China. Int J Earth Sci-Geol Rundsch, 98: 135-155

Zhu G, Jiang D, Zhang B, Chen Y. 2012. Destruction of the eastern North China Craton in a backarc setting: Evidence from crustal deformation kinematics. Gondwana Res, 22: 86-103 
Zhu R X, Fan H R, Li J W, Meng Q R, Li S R, Zeng Q D. 2015. Decratonic gold deposits. Sci China Earth Sci, 58: 1523-1537

Zhu R X, Yang J H, Wu F Y. 2012. Timing of destruction of the North China Craton. Lithos, 149: 51-60

Zhu R X, Zhang H, Zhu G, Meng Q, Fan H, Yang J, Wu F, Zhang Z,
Zheng T. 2017. Craton destruction and related resources. Int J Earth Sci-Geol Rundsch, 106: 2233-2257

Zhu R X, Zheng T Y. 2009. Destruction geodynamics of the North China craton and its Paleoproterozoic plate tectonics. Chin Sci Bull, 54: $3354-3366$

(责任编委: 姚华建) 